\title{
CONFORMATIONAL RIGIDIFICATION VIA DERIVATISATION \\ FACILITATES THE DETERMINATION OF ABSOLUTE CONFIGURATION USING CHIROPTICAL SPECTROSCOPY: A CASE STUDY OF THE CHIRAL ALCOHOL ENDO-BORNEOL
}

\author{
F.J. Devlin, P. J. Stephens* and P. Besse
}

\section{Supporting Information}

S1-S2 Contents

S3-S4 Figure S1: Comparison of B3PW91/TZ2P, B3LYP/TZ2P and experimental IR and VCD spectra of 5.

S5-S6 Table S1: Harmonic frequencies, dipole strengths and rotational strengths of 5.

S6-S7 Table S2: Assignment of Experimental IR and VCD peak frequencies of 5.

S8-S9 Figure S2: Comparison of B3PW91/TZ2P, B3LYP/TZ2P and experimental IR and VCD spectra of 4 .

S10-S11 Table S3: Harmonic frequencies, dipole strengths and rotational strengths of 4.

S11-S12 Table S4: Assignment of Experimental IR and VCD peak frequencies of 4.

S13-S14 Figure S3: Comparison of B3PW91/TZ2P, B3LYP/TZ2P and experimental IR and VCD spectra of 3.

S15-S16 Table S5: Harmonic frequencies, dipole strengths and rotational strengths of 3.

S16 Table S6: Assignment of Experimental IR and VCD peak frequencies of 3.

S17-S18 Figure S4: Comparison of B3PW91/TZ2P, B3LYP/TZ2P and experimental IR and VCD spectra of $\mathbf{1}$.

S19-S20 Table S7: Harmonic frequencies, dipole strengths and rotational strengths for the conformations of $\mathbf{1}$. 
S21 Table S8: Assignment of Experimental IR and VCD peak frequencies of 1.

S22 Figure S5: Concentration dependence of the IR spectrum of $\mathrm{CCl}_{4}$ solutions of 5 .

S23 Figure S6: Concentration dependence of the IR spectrum of $\mathrm{CCl}_{4}$ solutions of 4 .

S24 Figure S7: Concentration dependence of the IR spectrum of $\mathrm{CCl}_{4}$ solutions of 3 .

S25 Figure S8: Concentration dependence of the IR spectrum of $\mathrm{CCl}_{4}$ solutions of $\mathbf{1}$.

S26 Figure S9: Concentration dependence of the IR spectrum of $\mathrm{CCl}_{4}$ solutions of $\mathbf{1}$.

S27-S32 Cartesian coordinates of Conformations a - c of 1, 3a, 4a and 5a.

S33-S35 Synthesis and characterization. 


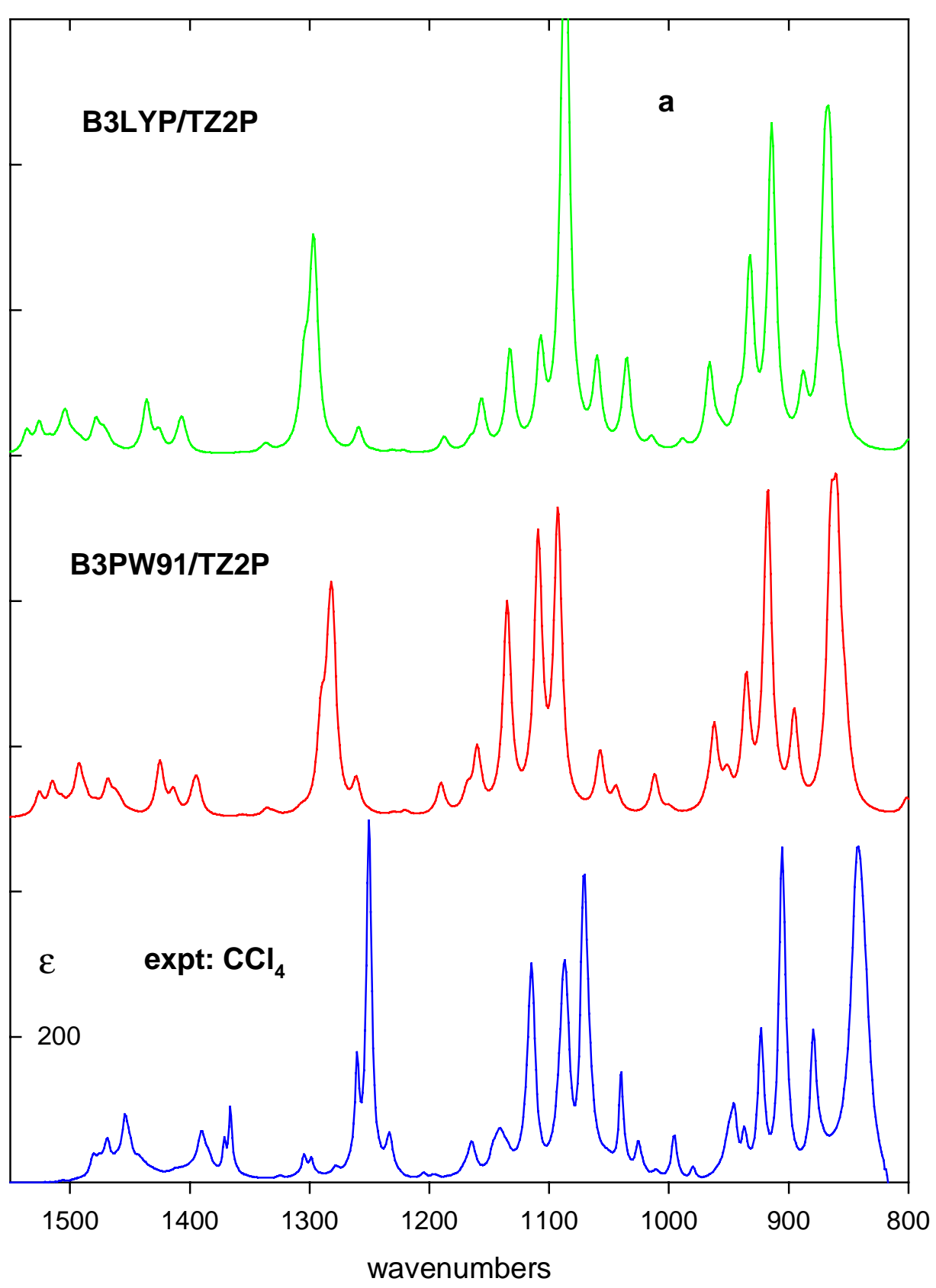




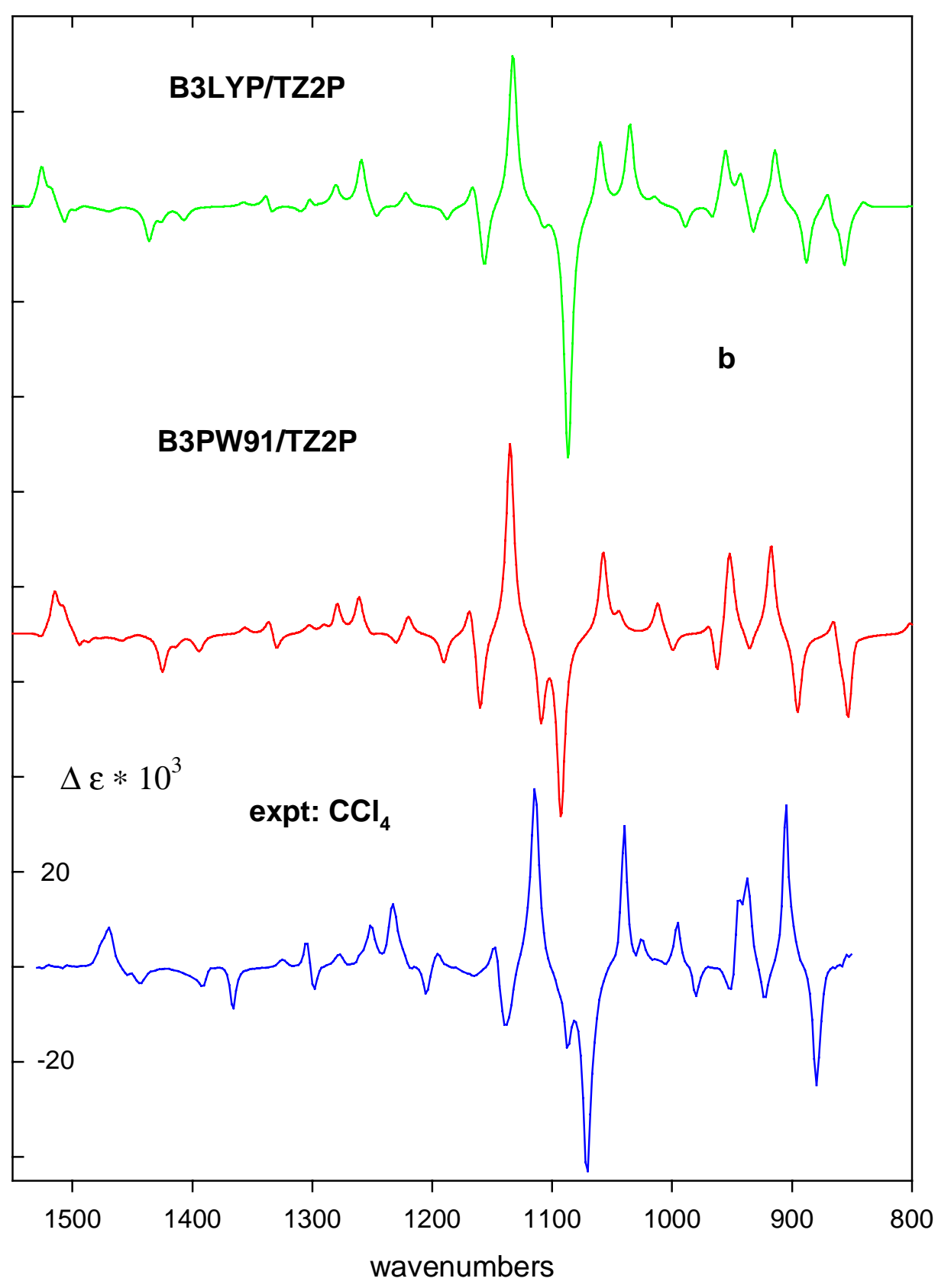

Figure S1 : Comparison of B3PW91/TZ2P, B3LYP/TZ2P and experimental IR (a) and VCD (b) spectra of 5. Calculations are for $1 \mathrm{R}, 2 \mathrm{~S}, 4 \mathrm{R}-5 \mathrm{a}$. Experimental spectra are from Figure 3 of the text. 
Table S1: Harmonic frequencies, dipole strengths and rotational strengths of $\mathbf{5}^{\mathrm{a}}$.

\begin{tabular}{|c|c|c|c|c|c|c|}
\hline \multirow[b]{2}{*}{ mode } & \multicolumn{3}{|c|}{ B3LYP/TZ2P } & \multicolumn{3}{|c|}{ B3PW91/TZ2P } \\
\hline & $v$ & $D$ & $R$ & $v$ & $D$ & $R$ \\
\hline 91 & 1536 & 21.8 & -2.0 & 1526 & 22.6 & -2.8 \\
\hline 90 & 1526 & 28.5 & 15.4 & 1515 & 31.6 & 15.9 \\
\hline 89 & 1517 & 8.9 & 5.6 & 1507 & 11.3 & 8.2 \\
\hline 88 & 1511 & 6.7 & -0.9 & 1500 & 6.5 & 0.9 \\
\hline 87 & 1506 & 15.0 & -8.5 & 1494 & 11.0 & -7.1 \\
\hline 86 & 1503 & 29.3 & 2.7 & 1492 & 39.8 & 2.6 \\
\hline 85 & 1497 & 9.6 & -1.3 & 1487 & 11.6 & -2.8 \\
\hline 84 & 1492 & 7.2 & 0.5 & 1480 & 6.6 & 0.1 \\
\hline 83 & 1490 & 2.5 & 0.3 & 1477 & 3.2 & -1.3 \\
\hline 82 & 1478 & 31.3 & -0.6 & 1469 & 32.9 & -0.6 \\
\hline 81 & 1472 & 15.4 & -0.8 & 1462 & 15.1 & -1.1 \\
\hline 80 & 1468 & 9.5 & -1.3 & 1458 & 10.0 & -1.8 \\
\hline 79 & 1461 & 0.9 & -0.3 & 1451 & 1.4 & -0.4 \\
\hline 78 & 1461 & 1.3 & 0.3 & 1449 & 1.3 & 0.0 \\
\hline 77 & 1457 & 1.0 & 0.3 & 1446 & 1.0 & 0.6 \\
\hline 76 & 1436 & 56.8 & -13.9 & 1425 & 59.5 & -15.5 \\
\hline 75 & 1426 & 20.5 & -4.6 & 1414 & 25.1 & -3.5 \\
\hline 74 & 1409 & 17.0 & -2.5 & 1397 & 18.3 & -2.0 \\
\hline 73 & 1406 & 28.5 & -3.6 & 1394 & 34.6 & -5.8 \\
\hline 72 & 1357 & 0.9 & 1.8 & 1356 & 1.9 & 2.9 \\
\hline 71 & 1338 & 6.7 & 8.1 & 1336 & 8.4 & 7.8 \\
\hline 70 & 1335 & 4.3 & -6.4 & 1330 & 3.5 & -8.7 \\
\hline 69 & 1310 & 3.1 & -2.3 & 1307 & 4.8 & 0.4 \\
\hline 68 & 1305 & 78.4 & -6.7 & 1303 & 0.7 & 3.4 \\
\hline 67 & 1303 & 11.0 & 9.1 & 1290 & 100.0 & 2.8 \\
\hline 66 & 1298 & 134.2 & -2.3 & 1283 & 146.1 & -3.4 \\
\hline 65 & 1296 & 133.0 & 1.2 & 1281 & 145.9 & -0.6 \\
\hline 64 & 1280 & 4.0 & 9.5 & 1280 & 7.6 & 15.7 \\
\hline 63 & 1259 & 30.5 & 22.7 & 1261 & 39.3 & 17.4 \\
\hline 62 & 1247 & 1.8 & -6.9 & 1250 & 1.3 & -2.0 \\
\hline 61 & 1231 & 3.2 & -0.9 & 1230 & 3.3 & -5.6 \\
\hline 60 & 1222 & 2.9 & 6.8 & 1220 & 5.9 & 9.5 \\
\hline 59 & 1188 & 20.2 & -6.8 & 1190 & 41.2 & -14.9 \\
\hline 58 & 1166 & 10.1 & 14.4 & 1168 & 26.4 & 18.8 \\
\hline 57 & 1157 & 67.9 & -34.6 & 1160 & 83.6 & -43.8 \\
\hline 56 & 1133 & 137.6 & 82.9 & 1135 & 287.9 & 104.3 \\
\hline 55 & 1107 & 134.4 & -8.5 & 1109 & 381.9 & -45.7 \\
\hline 54 & 1087 & 869.8 & -142.9 & 1093 & 427.9 & -100.9 \\
\hline 53 & 1060 & 124.0 & 38.6 & 1057 & 90.4 & 47.6 \\
\hline
\end{tabular}




\begin{tabular}{rrrrrrr}
52 & 1035 & 138.7 & 47.9 & 1044 & 35.6 & 10.0 \\
51 & 1014 & 18.3 & 4.4 & 1012 & 62.6 & 19.1 \\
50 & 989 & 14.3 & -13.0 & 1000 & 9.0 & -11.7 \\
49 & 966 & 135.5 & -11.1 & 968 & 12.5 & 9.6 \\
48 & 959 & 11.3 & 1.1 & 962 & 136.6 & -32.4 \\
47 & 956 & 12.4 & 35.3 & 952 & 24.4 & 45.0 \\
46 & 943 & 53.6 & 20.5 & 950 & 27.8 & 12.7 \\
45 & 932 & 303.5 & -21.2 & 935 & 212.3 & -14.1 \\
44 & 914 & 551.5 & 38.5 & 917 & 552.9 & 60.5 \\
43 & 888 & 102.5 & -39.2 & 895 & 162.0 & -54.6 \\
42 & 870 & 392.7 & 17.6 & 865 & 423.8 & 18.9 \\
41 & 866 & 413.6 & -11.5 & 860 & 432.1 & -12.8 \\
40 & 856 & 79.2 & -41.3 & 853 & 119.6 & -60.7 \\
39 & 841 & 3.1 & 5.6 & 848 & 14.1 & 10.0 \\
38 & 800 & 18.7 & 0.5 & 801 & 31.8 & 8.5 \\
\hline
\end{tabular}

${ }^{\mathrm{a}} v$ in $\mathrm{cm}^{-1}, D$ in $10^{-40} \mathrm{esu}^{2} \mathrm{~cm}^{2}, R$ in $10^{-44} \mathrm{esu}^{2} \mathrm{~cm}^{2} .(1 \mathrm{R}, 2 \mathrm{~S}, 4 \mathrm{R}) \mathrm{AC}$.

Table S2: Assignment of Experimental IR and VCD peak frequencies of $5^{\mathrm{a}}$.

\begin{tabular}{ccc}
\hline Assignment $^{\mathrm{b}}$ & $v_{\text {peak }}(\mathrm{IR})^{\mathrm{c}}$ & $\mathrm{v}_{\text {peak }}(\mathrm{VCD})^{\mathrm{c}}$ \\
\hline 91 & 1480 & \\
90 & 1469 & 1470 \\
87 & & 1445 \\
86 & 1454 & \\
76 & 1390 & 1392 \\
75 & 1384 & \\
74 & 1371 & \\
73 & 1367 & \\
$74 / 73$ & & 1366 \\
72 & 1325 & 1325 \\
71 & 1305 & 1305 \\
70 & 1299 & 1298 \\
68 & & 1278 \\
$69 / 68$ & 1278 & \\
67 & 1261 & \\
64 & & 1251 \\
$66-64$ & 1251 & \\
63 & 1234 & 1233 \\
62 & & 1218 \\
61 & 1204 & 1205 \\
60 & 1197 & 1196 \\
59 & 1165 & 1165
\end{tabular}




$\begin{array}{ccc}58 & 1147 & 1149 \\ 57 & 1142 & 1139 \\ 56 & 1115 & 1115 \\ 55 & 1087 & 1088 \\ 54 & 1071 & 1071 \\ 53 & 1040 & 1040 \\ 52 & 1026 & 1026 \\ 51 & 996 & 996 \\ 50 & 980 & 980 \\ 48 & & 953 \\ 49 / 48 & 946 & \\ 47 & & 944 \\ 46 & & 937 \\ 47 / 46 & 937 & \\ 45 & 923 & 923 \\ 44 & 906 & 906 \\ 43 & 880 & 880 \\ 42-39 & 842 & \end{array}$

${ }^{\mathrm{a}} \mathrm{In} \mathrm{CCl}_{4}$ solution.

${ }^{\mathrm{b}}$ Assignments based on B3PW91/TZ2P calculations.

${ }^{c} v_{\text {peak }}$ in $\mathrm{cm}^{-1}$. 


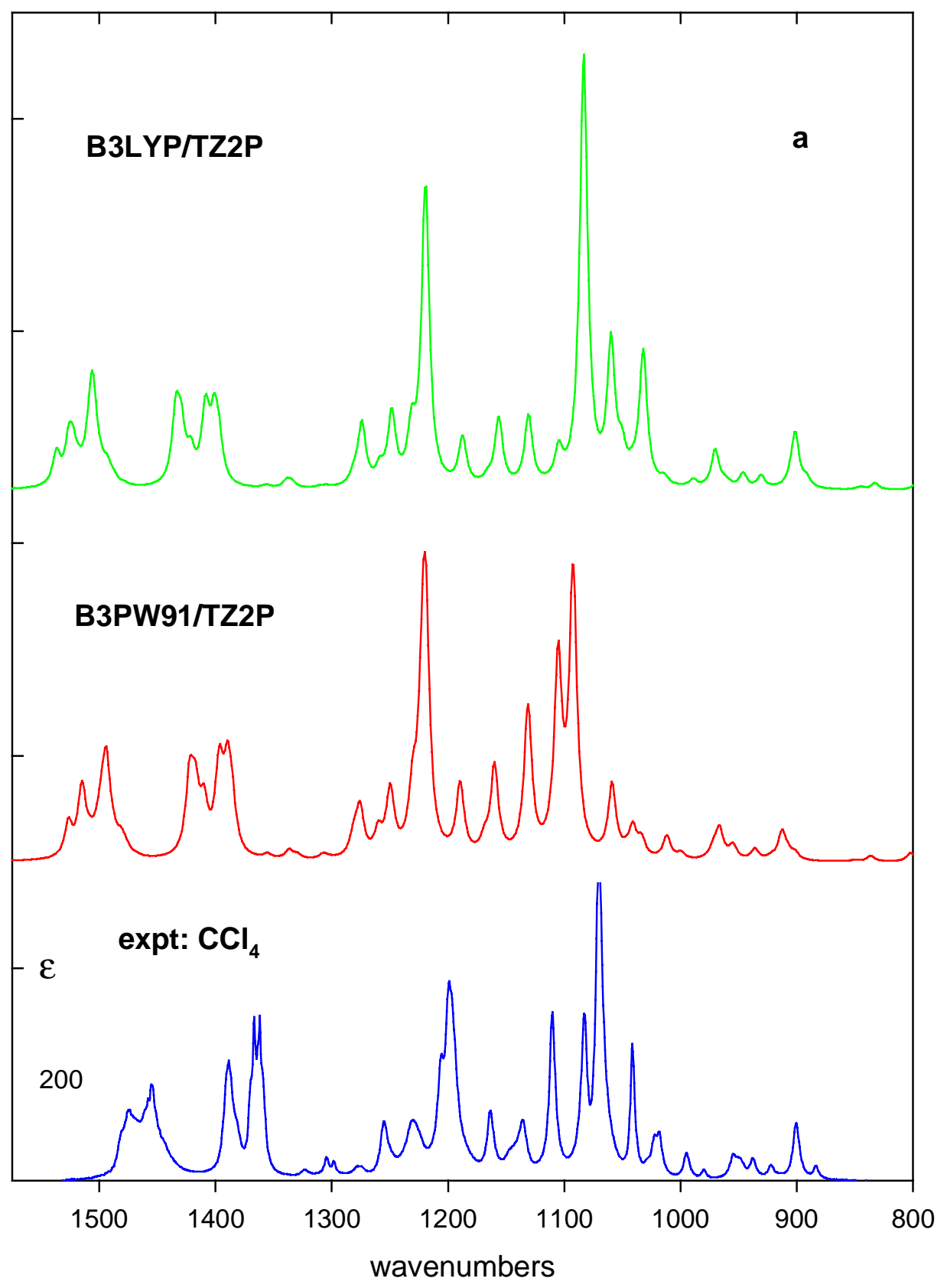




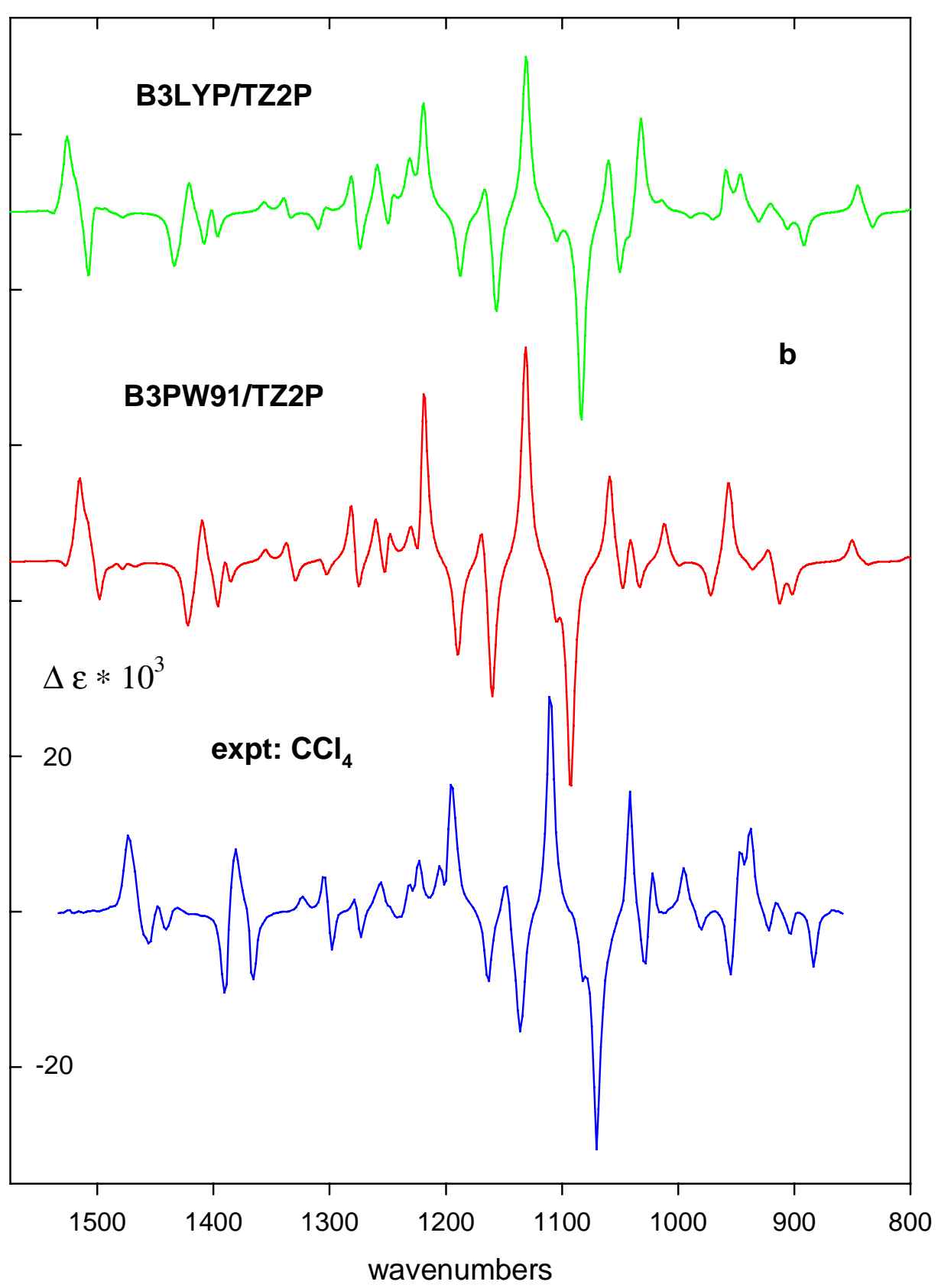

Figure S2 : Comparison of B3PW91/TZ2P, B3LYP/TZ2P and experimental IR (a) and VCD (b) spectra of 4. Calculations are for $1 \mathrm{R}, 2 \mathrm{~S}, 4 \mathrm{R}-4 \mathrm{a}$. Experimental spectra are from Figure 3 of the text. 
Table S3: Harmonic frequencies, dipole strengths and rotational strengths of $\mathbf{4}^{\mathrm{a}}$.

\begin{tabular}{|c|c|c|c|c|c|c|}
\hline \multirow[b]{2}{*}{ mode } & \multicolumn{3}{|c|}{ B3LYP/TZ2P } & \multicolumn{3}{|c|}{ B3PW91/TZ2P } \\
\hline & $v$ & $D$ & $R$ & v & $D$ & $R$ \\
\hline 91 & 1537 & 22.5 & -2.2 & 1526 & 23.5 & -3.1 \\
\hline 90 & 1526 & 27.2 & 16.9 & 1516 & 28.5 & 16.4 \\
\hline 89 & 1523 & 20.3 & 2.5 & 1514 & 21.1 & 3.8 \\
\hline 88 & 1518 & 6.7 & 4.9 & 1508 & 8.0 & 6.6 \\
\hline 87 & 1513 & 4.4 & 0.0 & 1503 & 6.8 & 0.9 \\
\hline 86 & 1508 & 24.1 & -8.9 & 1498 & 23.8 & -12.1 \\
\hline 85 & 1506 & 22.4 & -22.4 & 1495 & 10.6 & 1.7 \\
\hline 84 & 1505 & 32.8 & 12.3 & 1494 & 19.6 & -2.2 \\
\hline 83 & 1503 & 7.6 & 5.8 & 1493 & 35.6 & 1.7 \\
\hline 82 & 1498 & 7.4 & 0.5 & 1488 & 8.6 & -0.8 \\
\hline 81 & 1493 & 5.8 & -0.8 & 1482 & 6.5 & 1.7 \\
\hline 80 & 1493 & 5.5 & 1.9 & 1481 & 5.0 & -1.0 \\
\hline 79 & 1490 & 1.3 & -0.2 & 1478 & 3.2 & -2.8 \\
\hline 78 & 1487 & 3.7 & -0.1 & 1476 & 2.6 & 1.4 \\
\hline 77 & 1478 & 1.4 & -1.3 & 1467 & 1.2 & -1.1 \\
\hline 76 & 1434 & 52.6 & -12.4 & 1422 & 56.3 & -15.6 \\
\hline 75 & 1429 & 37.4 & -5.4 & 1417 & 42.6 & -5.5 \\
\hline 74 & 1421 & 20.7 & 10.4 & 1410 & 35.3 & 14.5 \\
\hline 73 & 1410 & 23.1 & -0.7 & 1398 & 23.6 & -0.1 \\
\hline 72 & 1408 & 35.2 & -8.8 & 1396 & 45.9 & -13.3 \\
\hline 71 & 1401 & 45.4 & 6.9 & 1390 & 57.3 & 6.7 \\
\hline 70 & 1397 & 28.3 & -8.7 & 1386 & 33.3 & -6.9 \\
\hline 69 & 1356 & 2.3 & 2.7 & 1355 & 3.6 & 3.3 \\
\hline 68 & 1339 & 5.8 & 5.3 & 1337 & 7.1 & 6.7 \\
\hline 67 & 1334 & 4.6 & -3.6 & 1330 & 3.5 & -7.0 \\
\hline 66 & 1310 & 1.5 & -6.0 & 1307 & 3.8 & 2.1 \\
\hline 65 & 1304 & 1.1 & 2.9 & 1303 & 0.8 & -4.9 \\
\hline 64 & 1281 & 8.6 & 13.6 & 1281 & 17.3 & 21.2 \\
\hline 63 & 1274 & 52.7 & -15.2 & 1276 & 39.8 & -15.1 \\
\hline 62 & 1259 & 13.3 & 14.8 & 1260 & 19.8 & 13.4 \\
\hline 61 & 1249 & 57.1 & -17.6 & 1251 & 8.9 & -22.7 \\
\hline 60 & 1247 & 4.4 & 14.7 & 1249 & 48.6 & 22.2 \\
\hline 59 & 1231 & 42.6 & 13.5 & 1230 & 48.4 & 8.7 \\
\hline 58 & 1222 & 1.5 & -6.2 & 1222 & 110.3 & -35.1 \\
\hline 57 & 1219 & 265.5 & 36.2 & 1219 & 184.1 & 73.2 \\
\hline 56 & 1188 & 43.0 & -20.7 & 1190 & 64.6 & -30.1 \\
\hline 55 & 1166 & 6.1 & 12.2 & 1169 & 12.4 & 16.6 \\
\hline 54 & 1156 & 63.1 & -34.6 & 1160 & 83.6 & -46.6 \\
\hline 53 & 1131 & 66.0 & 52.4 & 1131 & 141.4 & 72.4 \\
\hline
\end{tabular}




\begin{tabular}{rrrrrrr}
52 & 1105 & 30.6 & -8.5 & 1105 & 187.6 & -14.7 \\
51 & 1083 & 431.5 & -71.8 & 1092 & 279.3 & -76.8 \\
50 & 1060 & 141.6 & 23.4 & 1059 & 73.4 & 32.1 \\
49 & 1050 & 29.3 & -23.8 & 1047 & 4.4 & -15.3 \\
48 & 1041 & 9.0 & -9.9 & 1041 & 29.7 & 13.2 \\
47 & 1032 & 139.5 & 36.0 & 1034 & 17.8 & -11.6 \\
46 & 1014 & 7.7 & 3.3 & 1012 & 24.4 & 14.6 \\
45 & 989 & 7.7 & -2.4 & 1000 & 6.7 & -2.6 \\
44 & 970 & 42.2 & -3.4 & 972 & 11.5 & -13.7 \\
43 & 963 & 4.1 & -9.9 & 967 & 33.6 & -2.9 \\
42 & 961 & 0.1 & -0.2 & 957 & 4.0 & 28.3 \\
41 & 959 & 3.1 & 21.1 & 955 & 12.4 & 5.9 \\
40 & 946 & 16.3 & 14.0 & 950 & 0.1 & -1.7 \\
39 & 931 & 14.0 & -5.1 & 936 & 12.5 & -3.8 \\
38 & 921 & 0.6 & 4.4 & 922 & 3.5 & 7.6 \\
37 & 906 & 4.0 & -6.2 & 913 & 33.6 & -17.1 \\
36 & 901 & 66.7 & -0.3 & 907 & 6.9 & 0.3 \\
35 & 892 & 10.3 & -13.6 & 901 & 7.5 & -12.0 \\
34 & 845 & 3.2 & 12.7 & 850 & 1.6 & 9.6 \\
33 & 833 & 8.4 & -7.9 & 836 & 7.2 & -1.9 \\
\hline
\end{tabular}

${ }^{\mathrm{a}} v$ in $\mathrm{cm}^{-1}, D$ in $10^{-40} \mathrm{esu}^{2} \mathrm{~cm}^{2}, R$ in $10^{-44} \mathrm{esu}^{2} \mathrm{~cm}^{2} .(1 \mathrm{R}, 2 \mathrm{~S}, 4 \mathrm{R}) \mathrm{AC}$.

Table S4: Assignment of Experimental IR and VCD peak frequencies of $4^{\mathrm{a}}$.

\begin{tabular}{ccc}
\hline Assignment $^{\mathrm{b}}$ & $v_{\text {peak }}(\mathrm{IR})^{\mathrm{c}}$ & $v_{\text {peak }}(\mathrm{VCD})^{\mathrm{c}}$ \\
\hline 91 & 1480 & \\
$90-88$ & 1474 & 1474 \\
$86-83$ & 1455 & 1457 \\
81 & & 1449 \\
$81-80$ & 1445 & \\
$80 / 79$ & & 1442 \\
78 & 1389 & 1433 \\
$76 / 75$ & 1383 & 1391 \\
74 & 1367 & 1381 \\
$73 / 72$ & & 1366 \\
72 & 1362 & \\
$71 / 70$ & 1323 & 1324 \\
69 & 1305 & 1305 \\
68 & 1298 & 1298 \\
67 & & 1279 \\
66 & 1277 & \\
$66 / 65$ & &
\end{tabular}




\begin{tabular}{|c|c|c|}
\hline 65 & & 1274 \\
\hline 64 & & 1256 \\
\hline $64 / 63$ & 1255 & \\
\hline 63 & & 1240 \\
\hline 62 & & 1232 \\
\hline $62-60$ & 1230 & \\
\hline 60 & & 1223 \\
\hline 59 & 1206 & 1206 \\
\hline $58 / 57$ & 1199 & 1196 \\
\hline 56 & 1164 & 1164 \\
\hline 55 & 1146 & 1149 \\
\hline 54 & 1136 & 1136 \\
\hline 53 & 1110 & 1111 \\
\hline 52 & 1083 & 1082 \\
\hline 51 & 1070 & 1070 \\
\hline 50 & 1041 & 1042 \\
\hline 49 & 1029 & 1029 \\
\hline 48 & 1023 & 1022 \\
\hline 47 & 1019 & \\
\hline 46 & 995 & 995 \\
\hline 45 & 980 & 980 \\
\hline 44 & 955 & \\
\hline $44 / 43$ & & 955 \\
\hline 43 & 950 & \\
\hline 42 & & 946 \\
\hline $42 / 41$ & 938 & \\
\hline $41 / 40$ & & 938 \\
\hline 39 & 922 & 923 \\
\hline 38 & & 915 \\
\hline 37 & 901 & 904 \\
\hline $36 / 35$ & 884 & 884 \\
\hline
\end{tabular}

${ }^{\mathrm{a}} \mathrm{In} \mathrm{CCl}_{4}$ solution.

${ }^{\mathrm{b}}$ Assignments based on B3PW91/TZ2P calculations.

${ }^{\mathrm{c}} v_{\text {peak }}$ in $\mathrm{cm}^{-1}$. 


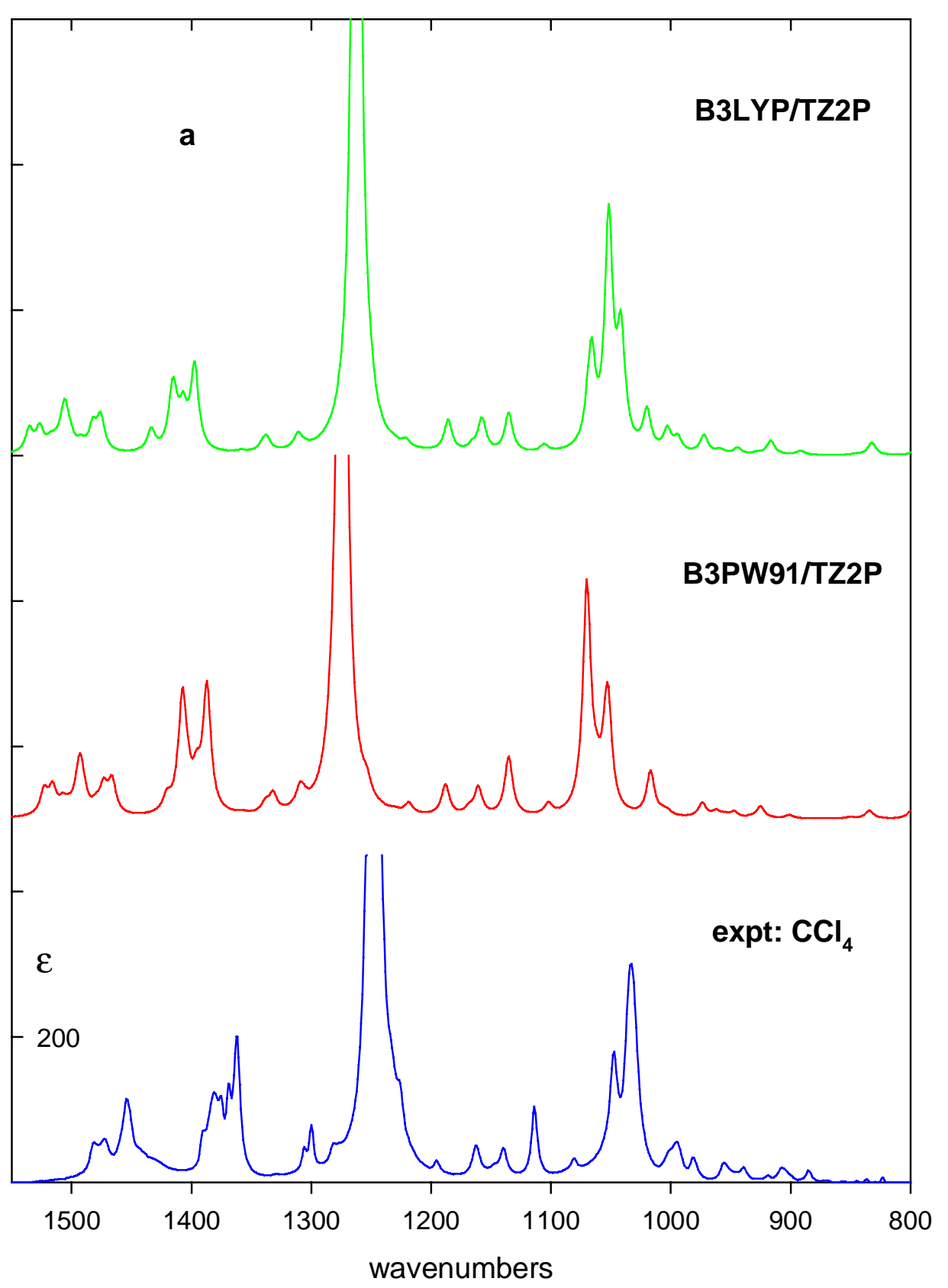




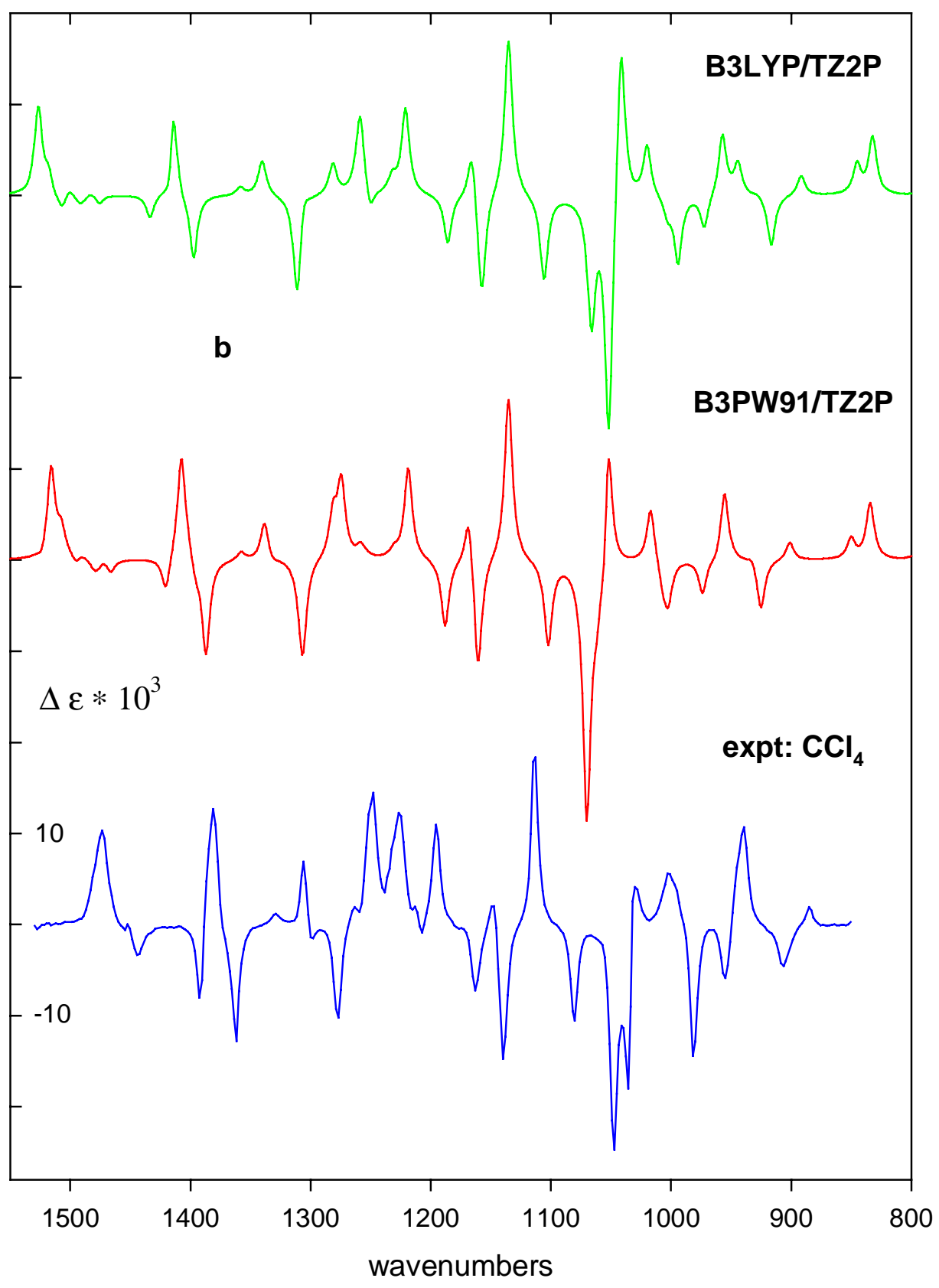

Figure S3 : Comparison of B3PW91/TZ2P, B3LYP/TZ2P and experimental IR (a) and VCD (b) spectra of 3. Calculations are for $1 \mathrm{R}, 2 \mathrm{~S}, 4 \mathrm{R}-3 \mathbf{a}$. Experimental spectra are from Figure 3 of the text. 
Table S5: Harmonic frequencies, dipole strengths and rotational strengths of $\mathbf{3}^{\mathrm{a}}$.

\begin{tabular}{|c|c|c|c|c|c|c|}
\hline \multirow[b]{2}{*}{ mode } & \multicolumn{3}{|c|}{ B3LYP/TZ2P } & \multicolumn{3}{|c|}{ B3PW91/TZ2P } \\
\hline & $v$ & $D$ & $R$ & $v$ & $D$ & $R$ \\
\hline 76 & 1781 & 556.0 & 31.7 & 1800 & 550.4 & 32.1 \\
\hline 75 & 1535 & 23.4 & -0.5 & 1523 & 24.8 & -2.0 \\
\hline 74 & 1527 & 24.1 & 18.2 & 1516 & 27.1 & 19.2 \\
\hline 73 & 1518 & 9.1 & 4.0 & 1507 & 11.5 & 5.5 \\
\hline 72 & 1513 & 6.9 & -1.1 & 1503 & 6.4 & 0.3 \\
\hline 71 & 1507 & 13.7 & -2.5 & 1495 & 9.2 & -1.2 \\
\hline 70 & 1505 & 37.3 & -0.9 & 1493 & 48.1 & -0.3 \\
\hline 69 & 1501 & 10.3 & 1.5 & 1490 & 11.7 & 0.8 \\
\hline 68 & 1492 & 8.0 & -1.4 & 1480 & 8.4 & -1.9 \\
\hline 67 & 1490 & 0.7 & -0.7 & 1476 & 1.1 & -1.3 \\
\hline 66 & 1482 & 25.7 & 0.8 & 1473 & 27.4 & 0.8 \\
\hline 65 & 1476 & 35.8 & -1.8 & 1466 & 35.7 & -2.6 \\
\hline 64 & 1434 & 23.8 & -5.1 & 1420 & 16.8 & -7.7 \\
\hline 63 & 1416 & 34.3 & -13.4 & 1408 & 125.1 & 23.3 \\
\hline 62 & 1415 & 42.4 & 28.2 & 1404 & 21.4 & 2.6 \\
\hline 61 & 1407 & 41.3 & -1.1 & 1396 & 34.0 & -3.4 \\
\hline 60 & 1397 & 95.9 & -14.8 & 1387 & 143.6 & -21.9 \\
\hline 59 & 1359 & 1.9 & 1.9 & 1358 & 2.0 & 2.0 \\
\hline 58 & 1340 & 7.4 & 8.0 & 1338 & 11.2 & 9.2 \\
\hline 57 & 1337 & 14.2 & 0.6 & 1332 & 21.5 & -1.1 \\
\hline 56 & 1311 & 17.4 & -24.7 & 1310 & 20.3 & -2.4 \\
\hline 55 & 1306 & 2.2 & 4.5 & 1307 & 8.2 & -22.2 \\
\hline 54 & 1281 & 1.2 & 7.7 & 1281 & 26.8 & 10.6 \\
\hline 53 & 1263 & 1316.5 & 1.6 & 1274 & 1291.3 & 18.7 \\
\hline 52 & 1259 & 59.6 & 19.7 & 1258 & 2.4 & 2.9 \\
\hline 51 & 1250 & 22.0 & -6.0 & 1254 & 18.5 & 0.5 \\
\hline 50 & 1232 & 2.6 & 4.0 & 1231 & 2.1 & 1.9 \\
\hline 49 & 1221 & 8.0 & 22.2 & 1219 & 13.0 & 23.9 \\
\hline 48 & 1186 & 41.7 & -13.0 & 1188 & 40.9 & -18.0 \\
\hline 47 & 1166 & 7.3 & 14.1 & 1168 & 8.3 & 15.1 \\
\hline 46 & 1158 & 45.5 & -28.7 & 1161 & 37.9 & -31.7 \\
\hline 45 & 1135 & 55.1 & 44.4 & 1135 & 82.6 & 45.9 \\
\hline 44 & 1106 & 9.9 & -24.1 & 1102 & 15.1 & -23.9 \\
\hline 43 & 1069 & 23.5 & -1.7 & 1070 & 337.1 & -75.5 \\
\hline 42 & 1066 & 130.8 & -35.1 & 1062 & 31.9 & -12.5 \\
\hline 41 & 1052 & 346.1 & -74.3 & 1055 & 45.9 & -20.0 \\
\hline 40 & 1042 & 167.3 & 52.3 & 1052 & 147.8 & 49.2 \\
\hline 39 & 1020 & 61.1 & 16.1 & 1017 & 69.4 & 17.3 \\
\hline 38 & 1003 & 34.1 & -5.5 & 1007 & 5.9 & -7.4 \\
\hline
\end{tabular}




\begin{tabular}{rrrrrrr}
37 & 994 & 22.3 & -21.1 & 1002 & 5.4 & -12.6 \\
36 & 972 & 29.5 & -10.9 & 974 & 23.1 & -11.2 \\
35 & 960 & 3.4 & -1.4 & 962 & 9.8 & -1.8 \\
34 & 957 & 3.6 & 21.2 & 955 & 4.5 & 23.2 \\
33 & 944 & 10.0 & 10.6 & 947 & 9.5 & 0.0 \\
32 & 928 & 2.9 & 1.1 & 933 & 1.8 & 2.3 \\
31 & 917 & 24.2 & -17.7 & 925 & 19.4 & -17.2 \\
30 & 892 & 6.9 & 7.2 & 901 & 5.5 & 6.4 \\
29 & 845 & 1.7 & 11.0 & 850 & 2.6 & 7.6 \\
28 & 832 & 24.0 & 21.8 & 834 & 15.1 & 21.2 \\
\hline
\end{tabular}

${ }^{\mathrm{a}} \mathrm{v}$ in $\mathrm{cm}^{-1}, D$ in $10^{-40} \mathrm{esu}^{2} \mathrm{~cm}^{2}, R$ in $10^{-44} \mathrm{esu}^{2} \mathrm{~cm}^{2} .(1 \mathrm{R}, 2 \mathrm{~S}, 4 \mathrm{R}) \mathrm{AC}$.

Table S6: Assignment of Experimental IR and VCD peak frequencies of $\mathbf{3}^{\mathrm{a}}$.

\begin{tabular}{ccc} 
Assignment $^{\mathrm{b}}$ & $v_{\text {peak }}(\mathrm{IR})^{\mathrm{c}}$ & $v_{\text {peak }}(\mathrm{VCD})^{\mathrm{c}}$ \\
\hline 74 & & 1474 \\
70 & 1454 & 1393 \\
64 & & 1381 \\
63 & 1361 & 1362 \\
60 & & 1306 \\
58 & & 1277 \\
55 & 1250 & \\
53 & 1167 & 1196 \\
49 & & 1163 \\
48 & 1144 & 1149 \\
47 & 1118 & 1140 \\
46 & & 1114 \\
45 & 1051 & 1081 \\
44 & & \\
43 & 1037 & 1048 \\
$43 / 42$ & & 1036 \\
$41 / 40$ & & 1030 \\
41 & 999 & 982 \\
40 & & 956 \\
39 & & 940 \\
37 & & 907 \\
36 & & \\
34 & &
\end{tabular}

${ }^{\mathrm{a}} \mathrm{In} \mathrm{CCl}_{4}$ solution.

b Assignments based on B3PW91/TZ2P calculations.

${ }^{\mathrm{c}} \mathrm{v}_{\text {peak }}$ in $\mathrm{cm}^{-1}$. 


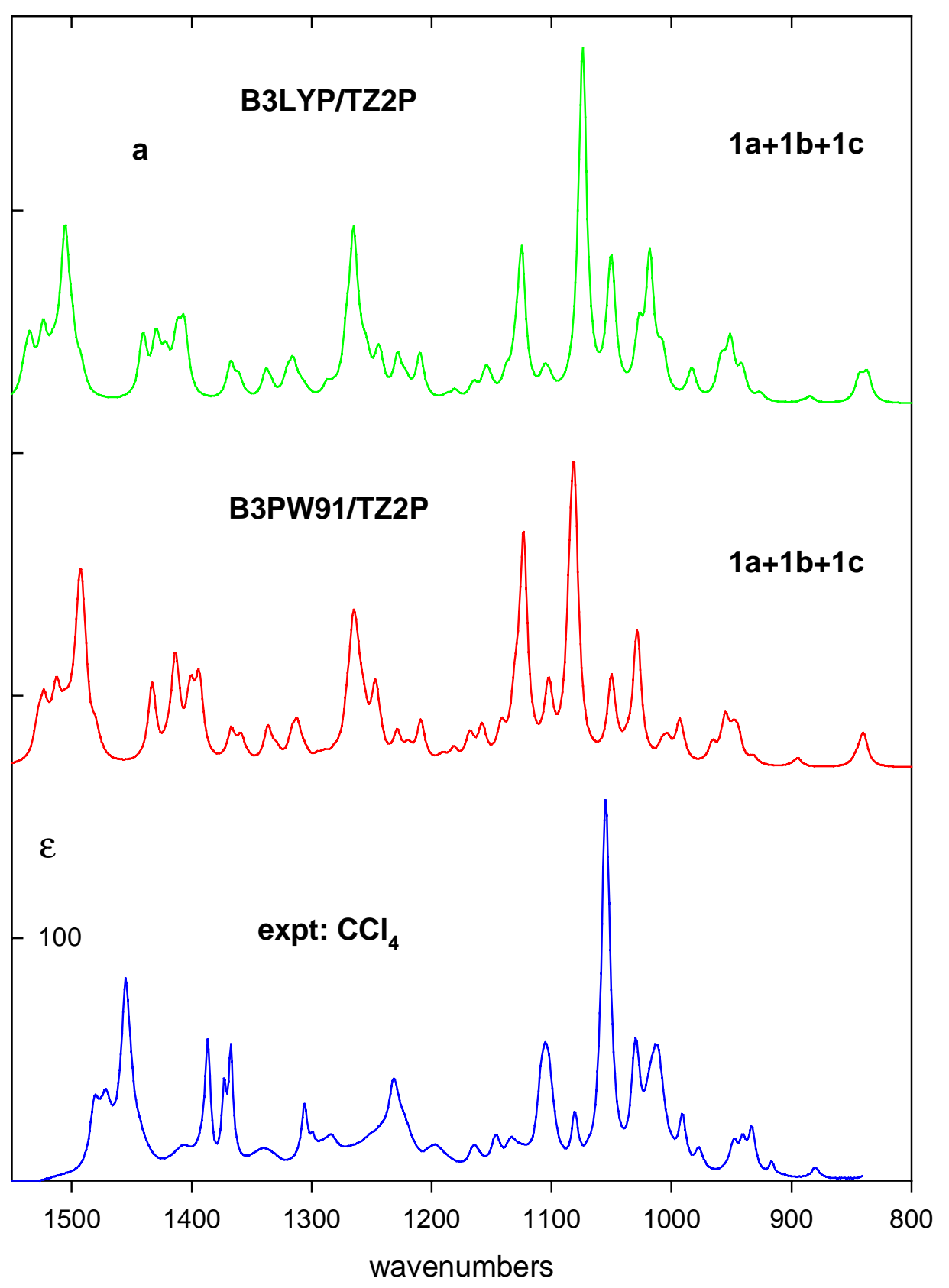




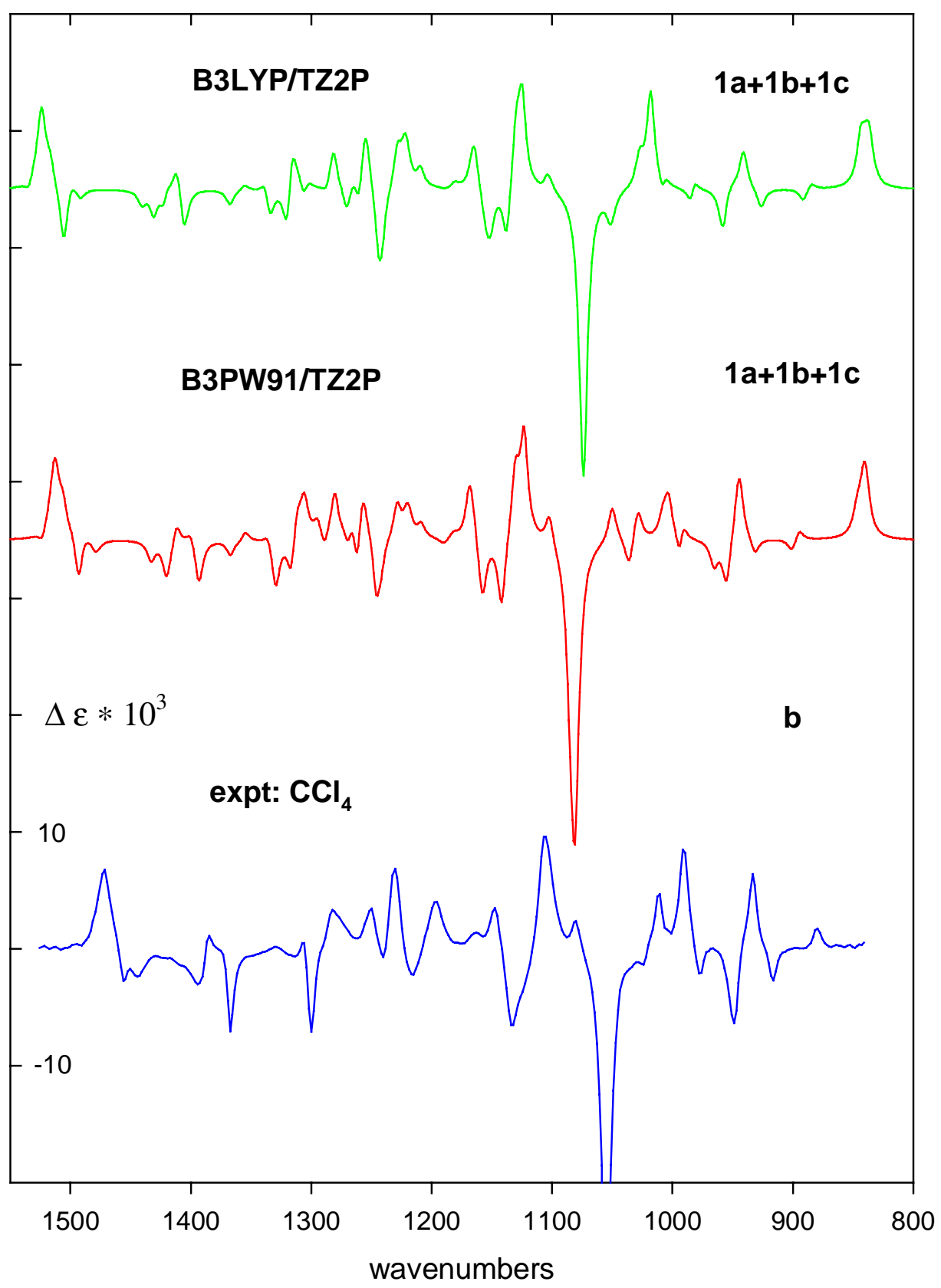

Figure S4 : Comparison of B3PW91/TZ2P, B3LYP/TZ2P and experimental IR (a) and VCD (b) spectra of $\mathbf{1}$. The conformationally-averaged calculated spectra are obtained from the spectra of 1R,2S,4R-1a, -1b and -1c using equilibrium populations calculated from relative free energies (Table 1 of the text). Experimental spectra are from Figure 3 of the text. 


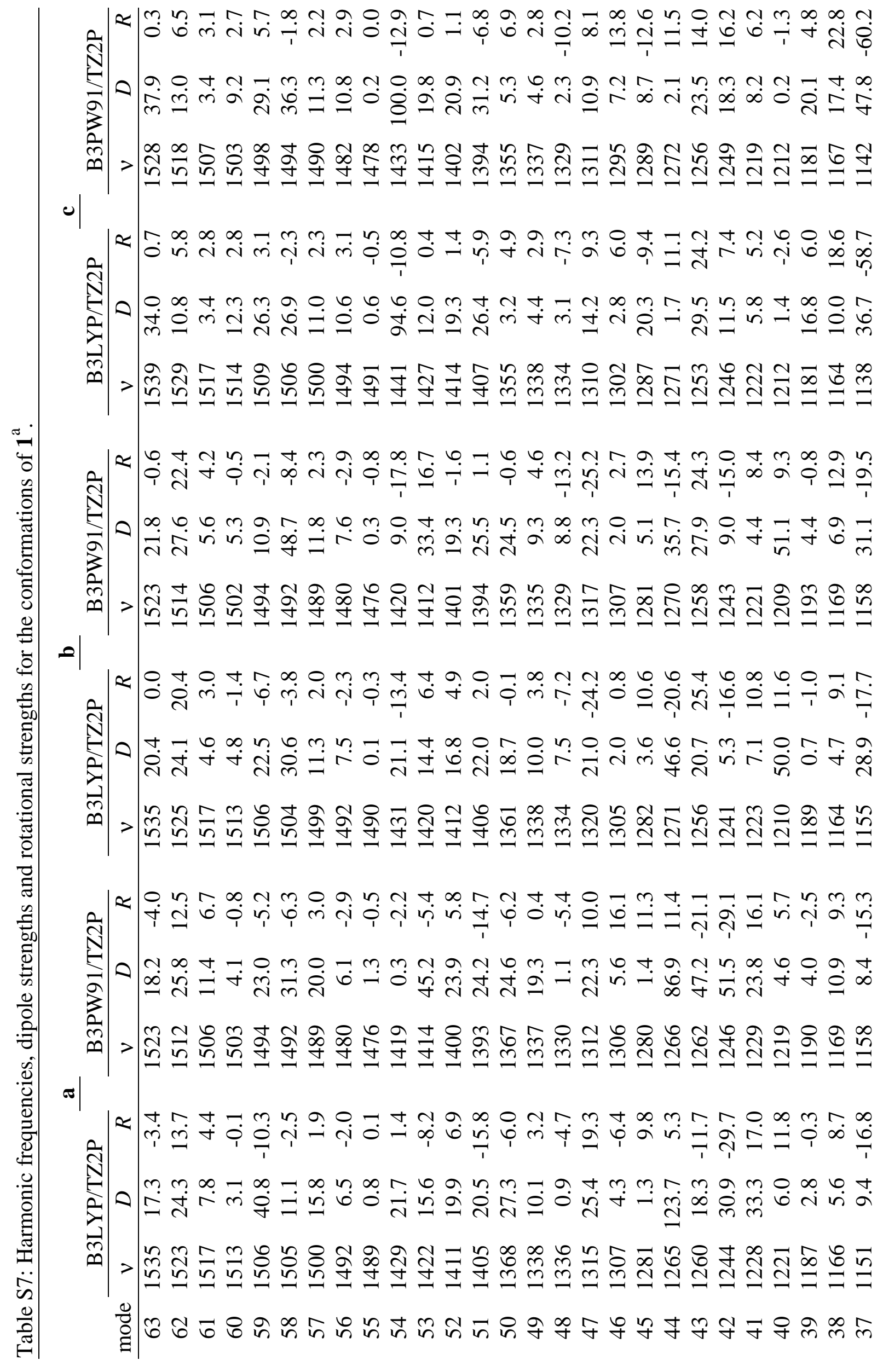




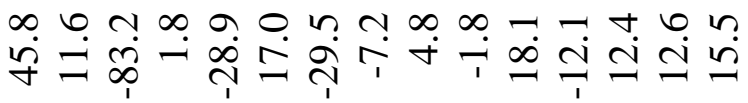
$\forall$ ․ லூ ம்

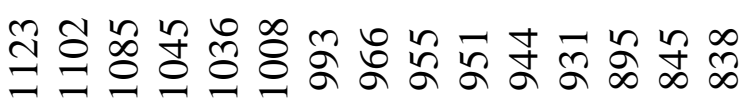

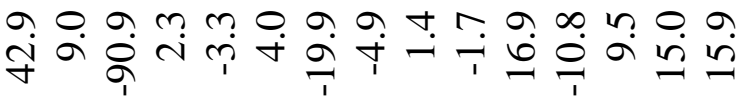

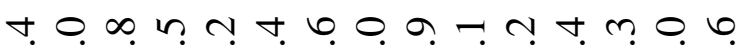

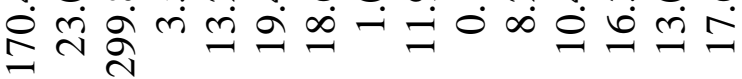

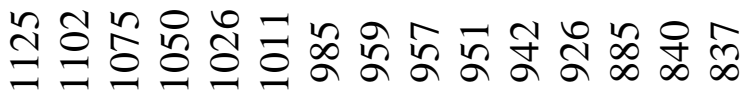

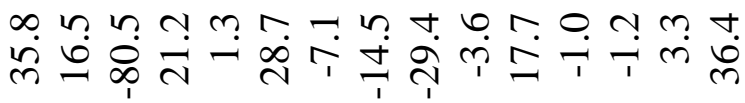
- a m 0 n $\infty$ b $\forall m+N-a N$ न

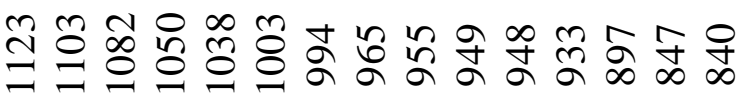

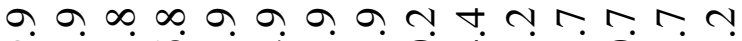

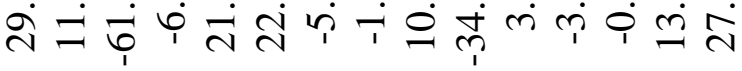

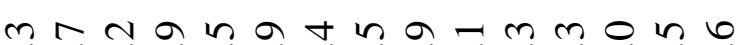

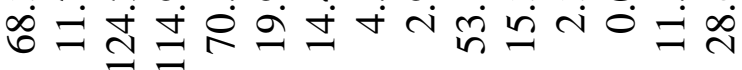

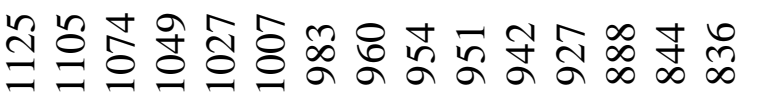

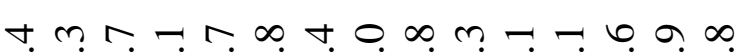
m ナ. .

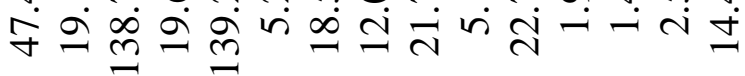

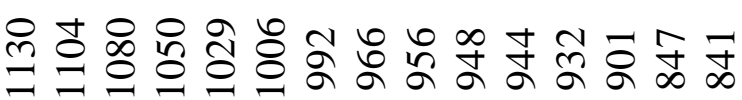
$\infty$ ๆ ヘึ ด ก ๆ

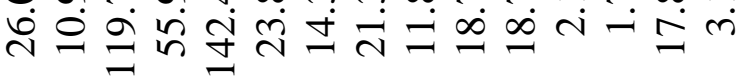

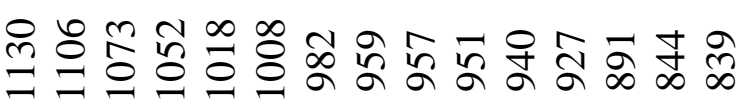


Table S8: Assignment of Experimental IR and VCD peak frequencies of $\mathbf{1}^{\mathrm{a}}$.

\begin{tabular}{|c|c|c|}
\hline Assignment ${ }^{\mathrm{b}}$ & $v_{\text {peak }}(\mathrm{IR})^{\mathrm{c}}$ & $v_{\text {peak }}(\mathrm{VCD})^{\mathrm{c}}$ \\
\hline 63 & 1481 & \\
\hline $62 / 61$ & 1472 & 1473 \\
\hline $60-57$ & 1455 & 1456 \\
\hline 56 & & 1445 \\
\hline $54 \mathrm{c}$ & 1407 & \\
\hline $54 \mathrm{~b}$ & & 1394 \\
\hline 53 & 1386 & \\
\hline $53 b$ & & 1385 \\
\hline 52 & 1373 & \\
\hline 51 & 1368 & 1367 \\
\hline $50 \mathrm{a} / 50 \mathrm{~b}$ & 1339 & \\
\hline $50 \mathrm{c}$ & & 1329 \\
\hline $49 / 48$ & 1306 & \\
\hline 48 & & 1301 \\
\hline 47 & 1284 & \\
\hline $47 / 46$ & & 1283 \\
\hline 45 & & 1251 \\
\hline $44-42$ & 1231 & \\
\hline 43 & & 1231 \\
\hline 42 & & 1216 \\
\hline $41 / 40$ & & 1197 \\
\hline $40 \mathrm{~b}$ & 1197 & \\
\hline 38 & & 1148 \\
\hline $37 b$ & 1134 & \\
\hline 37 & & 1134 \\
\hline $37 \mathrm{c}$ & & 1121 \\
\hline 36 & 1105 & 1106 \\
\hline 35 & 1081 & 1081 \\
\hline 34 & 1056 & 1056 \\
\hline 33 & 1030 & \\
\hline $32 a$ & 1014 & 1011 \\
\hline $31 b$ & & 991 \\
\hline 31 & 991 & \\
\hline 30 & 977 & \\
\hline $30 c$ & & 978 \\
\hline $29-27$ & & 950 \\
\hline 28 & 941 & \\
\hline $25 \mathrm{c}$ & & 917 \\
\hline $24 c$ & 881 & 880 \\
\hline
\end{tabular}

${ }^{\mathrm{a}} \mathrm{In}_{\mathrm{CCl}}$ solution.

${ }^{\mathrm{b}}$ Assignments based on B3PW91/TZ2P calculations.

${ }^{\mathrm{c}} v_{\text {peak }}$ in $\mathrm{cm}^{-1}$. 


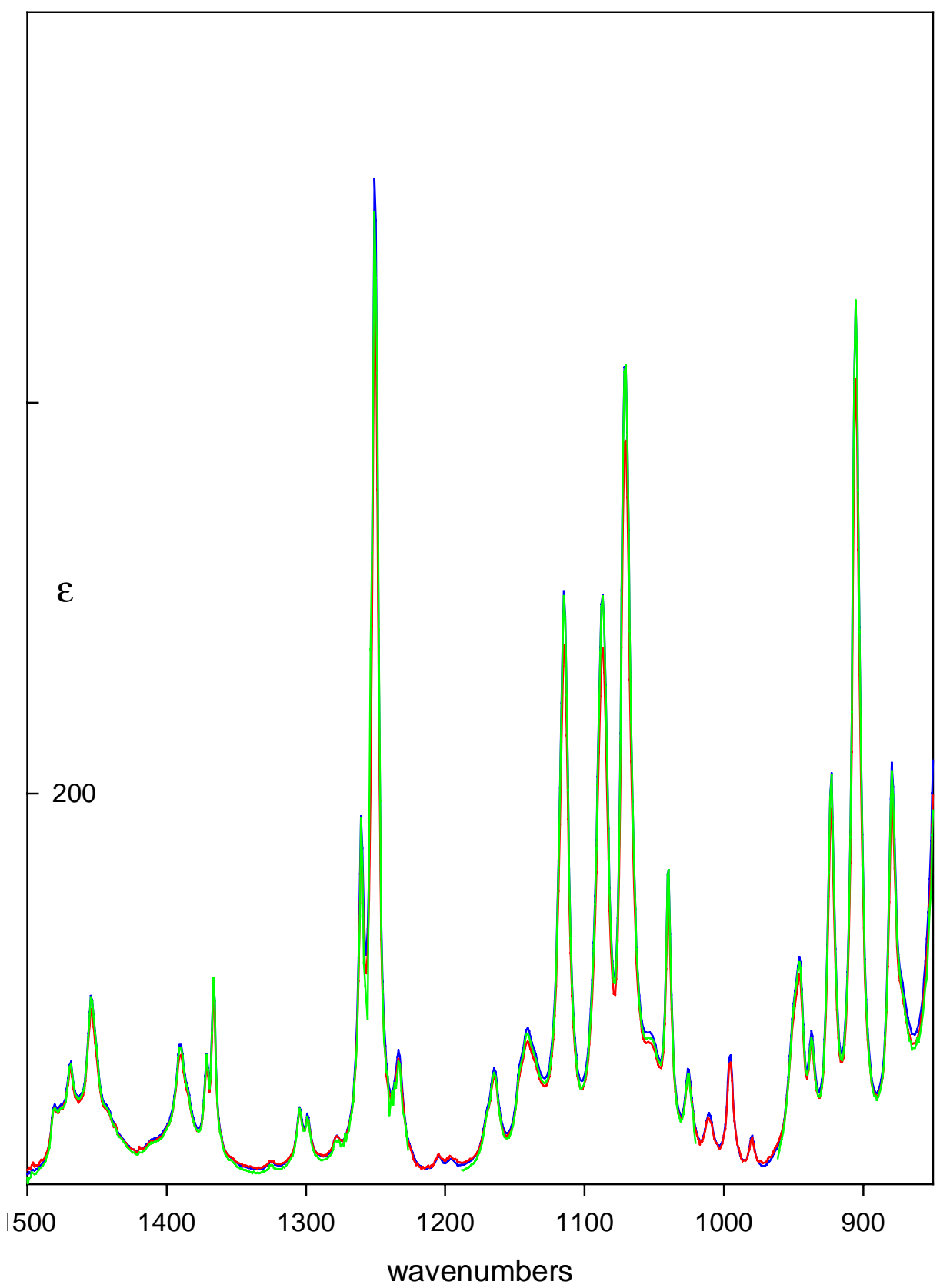

Figure S5 : Concentration dependence of the IR spectrum of $\mathrm{CCl}_{4}$ solutions of 5 . $0.148 \mathrm{M}$, pathlength $109 \mu$ (blue); $0.0148 \mathrm{M}$, pathlength $597 \mu$ (red); $0.0037 \mathrm{M}$, pathlength $1637 \mu$ (green). 


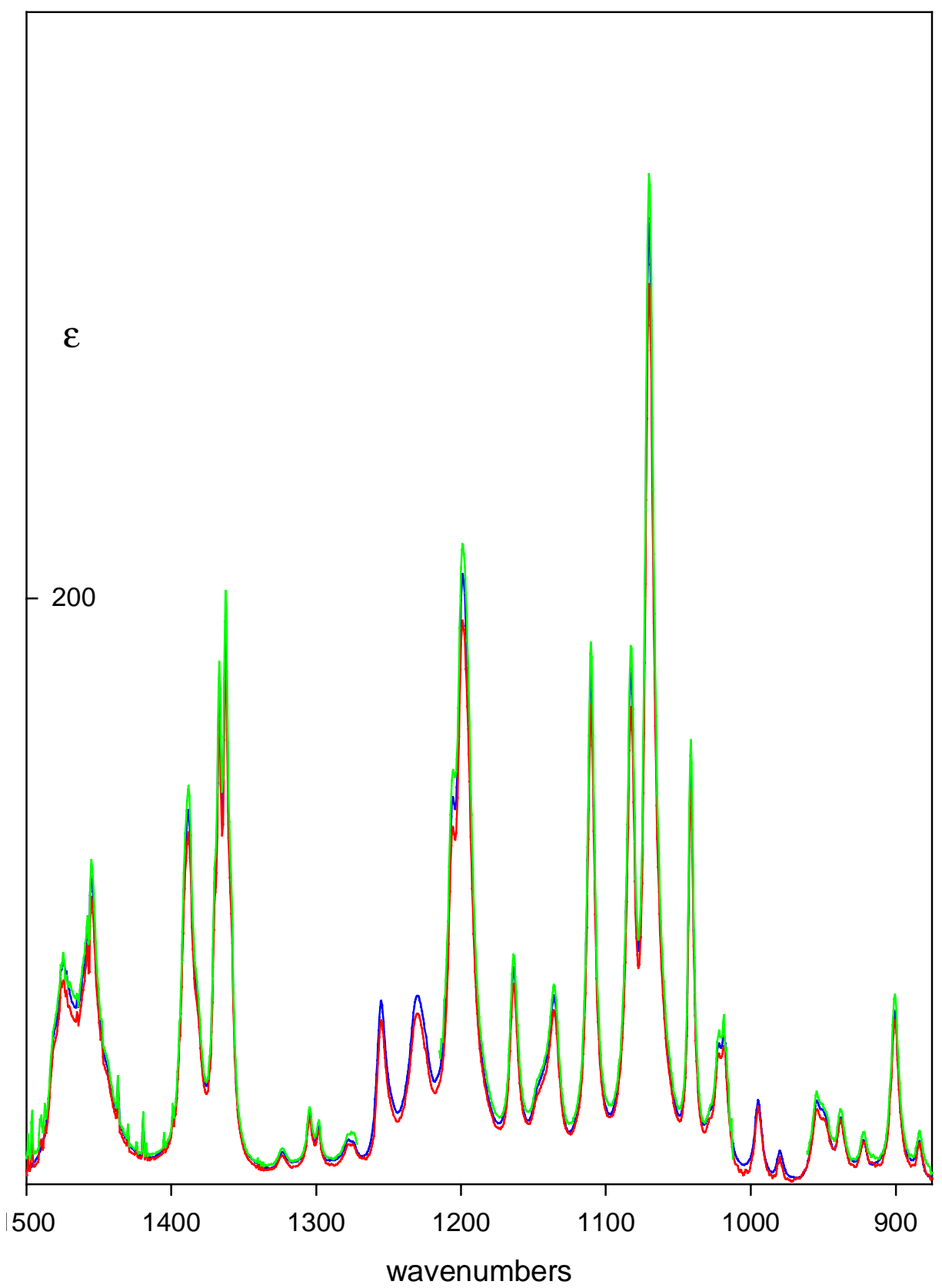

Figure S6 : Concentration dependence of the IR spectrum of $\mathrm{CCl}_{4}$ solutions of 4. $0.1536 \mathrm{M}$, pathlength $109 \mu$ (blue); $0.01536 \mathrm{M}$, pathlength $597 \mu$ (red); $0.00384 \mathrm{M}$, pathlength $1637 \mu$ (green). 


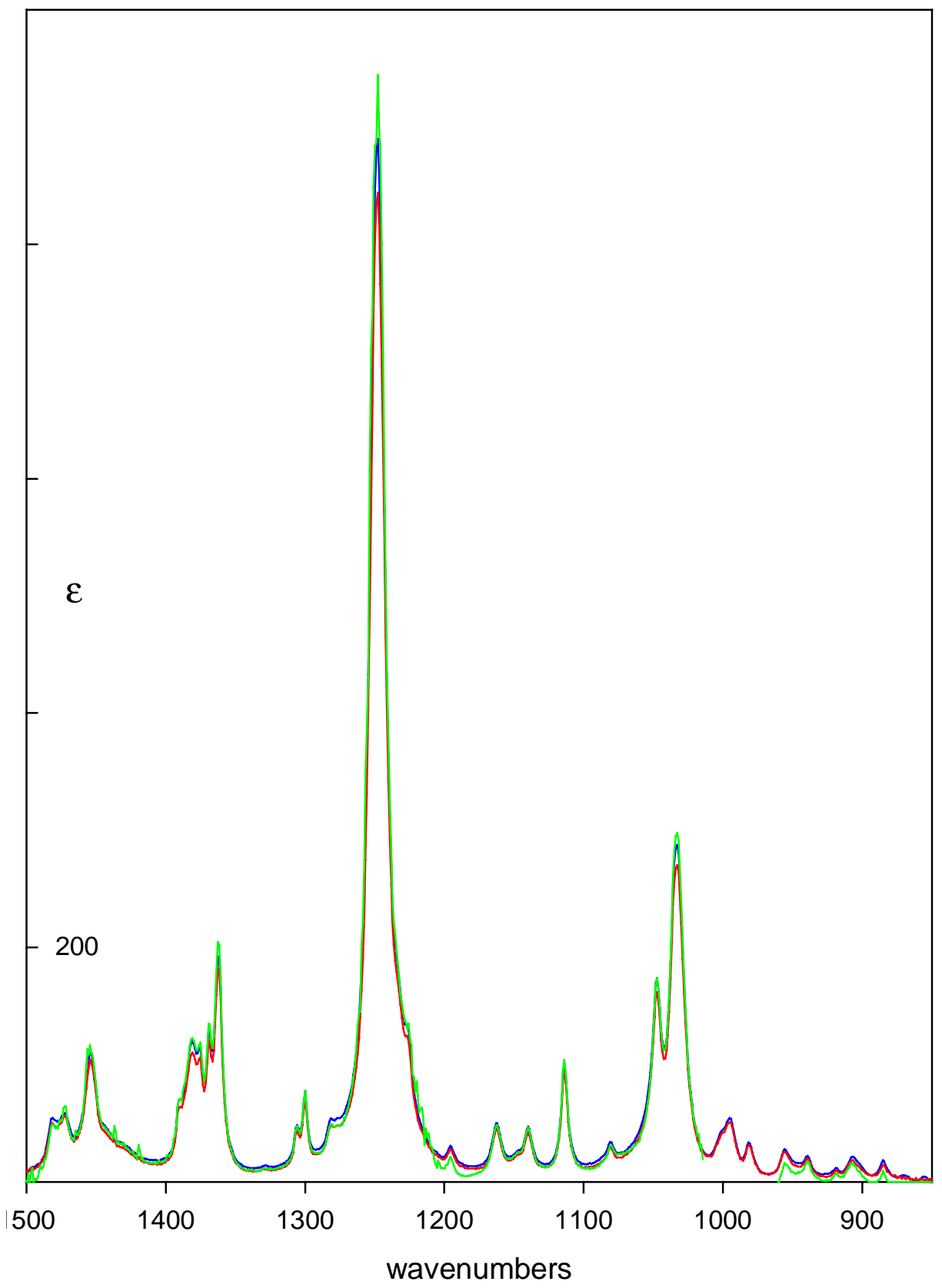

Figure S7 : Concentration dependence of the IR spectrum of $\mathrm{CCl}_{4}$ solutions of 3. $0.180 \mathrm{M}$, pathlength $109 \mu$ (blue); $0.0180 \mathrm{M}$, pathlength $597 \mu$ (red); $0.0045 \mathrm{M}$, pathlength $1637 \mu$ (green). 


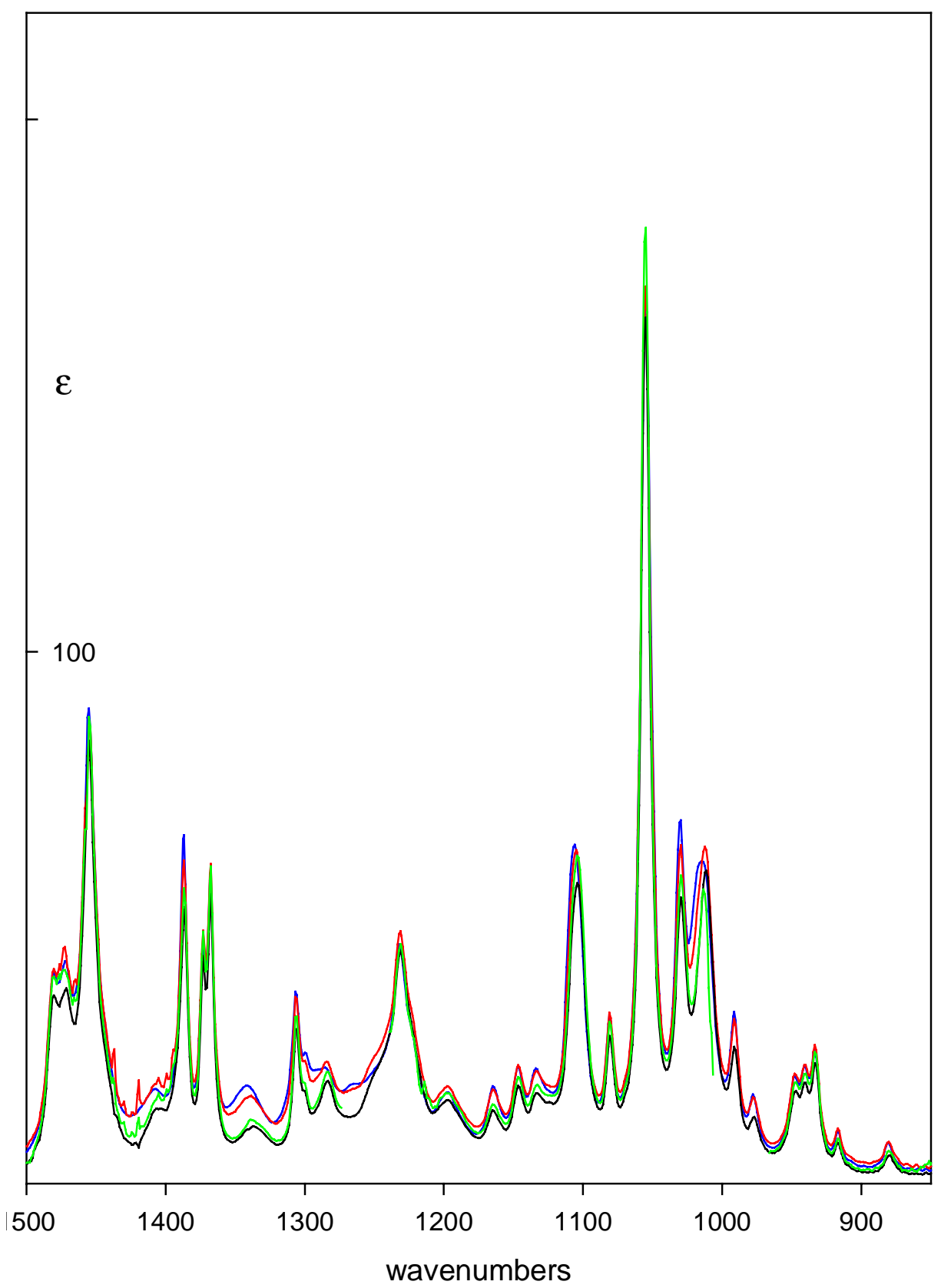

Figure S8 : Concentration dependence of the IR spectrum of $\mathrm{CCl}_{4}$ solutions of 1. $0.3062 \mathrm{M}$, pathlength $239 \mu$ (blue); $0.1531 \mathrm{M}$, pathlength $239 \mu$ (red); $0.03062 \mathrm{M}$, pathlength $597 \mu$ (black); $0.00766 \mathrm{M}$, pathlength $1637 \mu$ (green). 


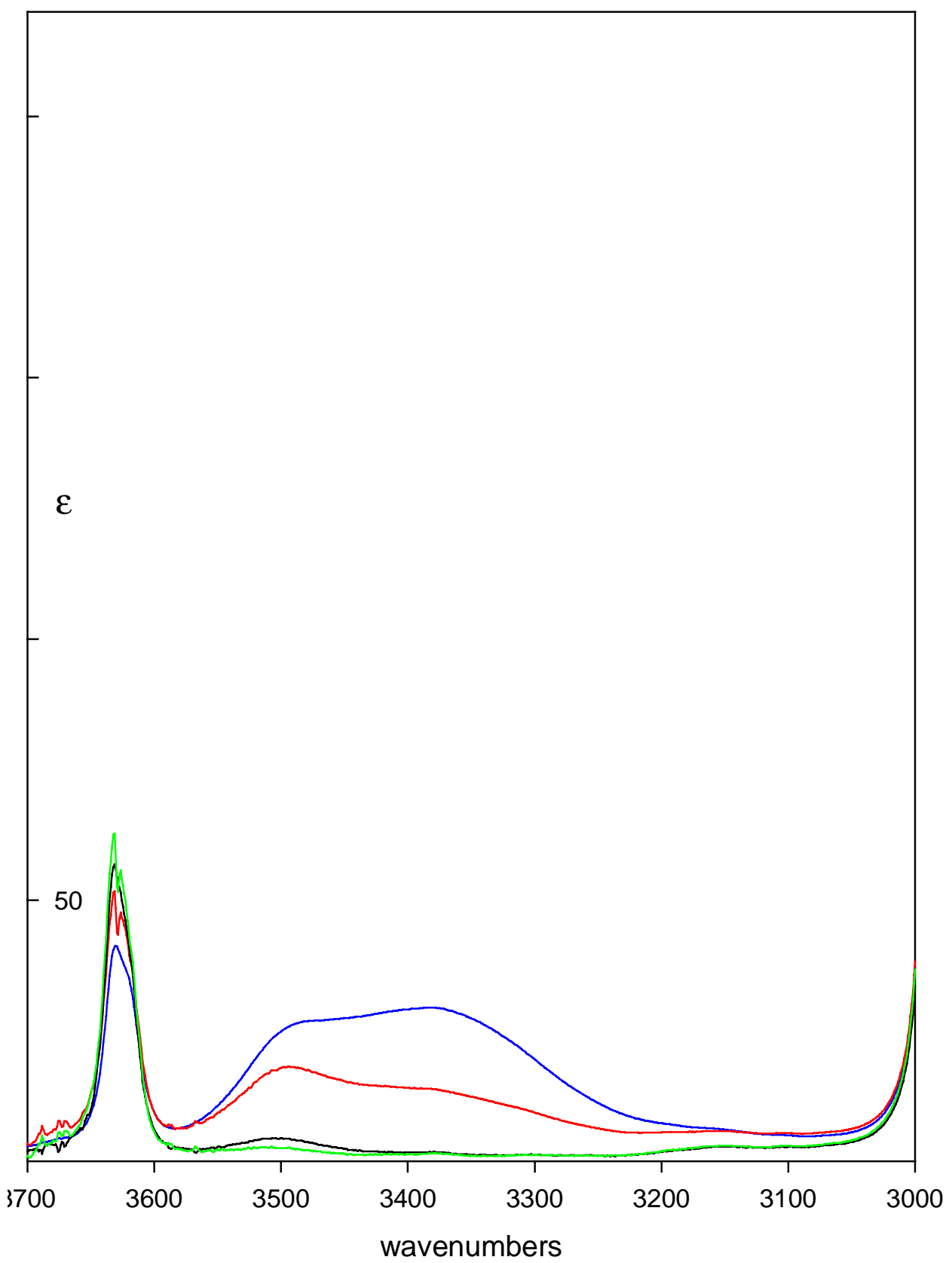

Figure S9 : Concentration dependence of the IR spectrum of $\mathrm{CCl}_{4}$ solutions of 1. $0.3062 \mathrm{M}$, pathlength $239 \mu$ (blue); $0.1531 \mathrm{M}$, pathlength $239 \mu$ (red); $0.03062 \mathrm{M}$, pathlength $597 \mu$ (black); $0.00766 \mathrm{M}$, pathlength $1637 \mu$ (green). 
Borneol derivative 1 conformer a $(1 R, 2 S, 4 R)$-configuration B3PW91/TZ2P optimized geometry [E=-467.129113747 hartrees] Cartesian coordinates (Angstroms)
$-0.764297$
$-0.014108$
$-0.062855$
$-0.745964$
$-0.033177$
1.479302
0.757610
$-0.144492$
1.837601
1.434930
$-0.128141$
1.280740
1.296017
$-0.207397$
1.366686
0.459169
0.445588
$-0.942892$
$-0.823341$
2.490152
$-1.708075$
2.585505
1.469476
$-1.317871$
$-0.880277$
$-1.201195$
0.871592
0.684332
1.098916
$-1.063177$
$-0.494809$
1. 485681
2.055398
$-0.347741$
$-1.342672$
$-0.312358$
0.356891
$-0.984894$
$-1.452302$
$-0.008450$
$-1.590931$
$-2.392994$
$-2.937364$
$-2.490250$
$-2.903074$
$-0.095629$
$-0.496614$
$-0.415869$
0.121889
$-0.237847$
$-1.581259$
1.860202
1.879064
2.458655
2.382863
0.462417
$-0.947328$
0.655353
$-0.672184$
$-0.376542$
$-1.763896$
$-0.339272$
$-1.899615$
$-2.488796$
$-2.336041$
$-2.034308$
0.036234
1.208390
$-0.053613$
$-0.070041$
1.071371
$-0.586938$ 
Borneol derivative 1 conformer $b \quad(1 R, 2 S, 4 R)$-configuration B3PW91/TZ2P optimized geometry [E=-467.128609957 hartrees] Cartesian coordinates (Angstroms)

$\begin{array}{rrr}-0.764777 & -0.037791 & -0.061976 \\ -0.736994 & -0.057163 & 1.481999 \\ 0.771399 & -0.112172 & 1.832226 \\ 1.442601 & -0.082668 & 0.450333 \\ 1.242666 & 1.332732 & -0.112156 \\ -0.261243 & 1.354605 & -0.494672 \\ 0.473220 & -0.927964 & -0.417498 \\ -1.021752 & 2.376144 & 0.141129 \\ -0.779074 & 3.217044 & -0.250609 \\ -0.384000 & 1.457482 & -1.577369 \\ -1.271792 & -0.929976 & 1.858423 \\ -1.230859 & 0.822703 & 1.888952 \\ 1.087014 & 0.732456 & 2.445907 \\ 1.026094 & -1.018173 & 2.382965 \\ 2.480547 & -0.419269 & 0.451158 \\ 1.880613 & 1.528325 & -0.975225 \\ 1.456646 & 2.098993 & 0.634380 \\ -2.103735 & -0.411307 & -0.663136 \\ -2.423750 & -1.403162 & -0.338017 \\ -2.070308 & -0.408224 & -1.755117 \\ -2.870318 & 0.301766 & -0.355300 \\ 0.843189 & -0.962585 & -1.903473 \\ 0.067639 & -1.468891 & -2.481995 \\ 0.994578 & 0.019319 & -2.348252 \\ 1.767742 & -1.527325 & -2.043151 \\ 0.342781 & -2.381475 & 0.039444 \\ 1.296479 & -2.899891 & -0.084382 \\ 0.045221 & -2.485938 & 1.080677 \\ -0.393103 & -2.911668 & -0.568627\end{array}$


Borneol derivative 1 conformer $c \quad(1 R, 2 S, 4 R)$-configuration B3PW91/TZ2P optimized geometry [E=-467.128366435 hartrees] Cartesian coordinates (Angstroms)
$-0.684025$ 
Borneol derivative 3 conformer a (1R,2S,4R)-configuration B3PW91/TZ2P optimized geometry [E=-619.790590973 hartrees] Cartesian coordinates (Angstroms)

$\begin{array}{rrrr}6 & -0.692737 & -0.267567 & 0.732225 \\ 6 & -0.791405 & -1.710928 & 0.190398 \\ 6 & -1.284733 & -1.535330 & -1.268589 \\ 6 & -1.383837 & -0.009268 & -1.412207 \\ 6 & 0.053016 & 0.533028 & -1.442687 \\ 6 & 0.501271 & 0.403920 & 0.030411 \\ 6 & -1.881027 & 0.434003 & -0.010764 \\ 8 & 1.692347 & -0.390446 & 0.204814 \\ 6 & 2.862317 & 0.232448 & -0.023455 \\ 6 & 4.019694 & -0.700022 & 0.198830 \\ 1 & 4.952027 & -0.156590 & 0.079149 \\ 1 & 3.965460 & -1.139985 & 1.194452 \\ 1 & 3.975926 & -1.517724 & -0.521675 \\ 8 & 2.956439 & 1.383603 & -0.365280 \\ 1 & 0.712300 & 1.378640 & 0.465450 \\ 1 & -1.496953 & -2.289036 & 0.788167 \\ 1 & 0.168088 & -2.220651 & 0.253397 \\ 1 & -0.593291 & -1.960072 & -1.997383 \\ 1 & -2.249423 & -2.018001 & -1.427386 \\ 1 & -1.999145 & 0.321551 & -2.249846 \\ 1 & 0.109602 & 1.567566 & -1.777686 \\ 1 & 0.696516 & -0.052525 & -2.099742 \\ 6 & -0.647060 & -0.170581 & 2.243082 \\ 1 & -1.527315 & -0.630186 & 2.696200 \\ 1 & -0.595834 & 0.867468 & 2.578702 \\ 1 & 0.231110 & -0.686451 & 2.636390 \\ 6 & -1.949512 & 1.953817 & 0.168303 \\ 1 & -2.187067 & 2.209578 & 1.203014 \\ 1 & -1.033176 & 2.477221 & -0.098027 \\ 1 & -2.747936 & 2.363631 & -0.454140 \\ 6 & -3.256543 & -0.118233 & 0.365186 \\ 1 & -4.017712 & 0.292390 & -0.302007 \\ 1 & -3.324469 & -1.202841 & 0.311388 \\ 1 & -3.525656 & 0.179036 & 1.380894\end{array}$


Borneol derivative 4 conformer a (1R,2S,4R)-configuration B3PW91/TZ2P optimized geometry [E=-624.374121640 hartrees] Cartesian coordinates (Angstroms)

$\begin{array}{rrrr}6 & 0.939868 & 0.518471 & -0.530974 \\ 6 & 1.155838 & -0.517519 & -1.656745 \\ 6 & 1.761772 & -1.744235 & -0.929458 \\ 6 & 1.778889 & -1.292845 & 0.538880 \\ 6 & 0.317382 & -1.254270 & 1.011133 \\ 6 & -0.254051 & 0.012368 & 0.313182 \\ 6 & 2.137579 & 0.211848 & 0.427384 \\ 8 & -1.386820 & -0.234094 & -0.501408 \\ 6 & -2.690135 & 0.040889 & 0.039024 \\ 6 & -2.903589 & 1.547607 & 0.193141 \\ 1 & -3.922953 & 1.754485 & 0.523256 \\ 1 & -2.227313 & 1.983280 & 0.929701 \\ 1 & -2.742058 & 2.051230 & -0.760163 \\ 6 & -3.644733 & -0.510843 & -1.013317 \\ 1 & -3.492364 & -1.583188 & -1.137753 \\ 1 & -4.681895 & -0.337525 & -0.723697 \\ 1 & -3.467677 & -0.027202 & -1.974266 \\ 6 & -2.912882 & -0.673534 & 1.371939 \\ 1 & -2.236005 & -0.307510 & 2.145038 \\ 1 & -3.932770 & -0.504843 & 1.720949 \\ 1 & -2.762451 & -1.747914 & 1.263515 \\ 1 & -0.505269 & 0.776341 & 1.052193 \\ 1 & 1.833131 & -0.120799 & -2.414353 \\ 1 & 0.214903 & -0.745286 & -2.153481 \\ 1 & 1.161465 & -2.645335 & -1.061011 \\ 1 & 2.764940 & -1.973394 & -1.290737 \\ 1 & 2.437914 & -1.882068 & 1.178685 \\ 1 & 0.235132 & -1.191410 & 2.097117 \\ 1 & -0.232506 & -2.139891 & 0.693377 \\ 6 & 0.779153 & 1.940129 & -1.025695 \\ 1 & 1.645276 & 2.257568 & -1.609809 \\ 1 & 0.652752 & 2.644725 & -0.200426 \\ 1 & -0.099258 & 2.023078 & -1.668089 \\ 6 & 2.104180 & 0.958414 & 1.763813 \\ 1 & 2.219010 & 2.033477 & 1.608799 \\ 1 & 1.191402 & 0.804140 & 2.336001 \\ 1 & 2.937109 & 0.634696 & 2.392130 \\ 6 & 3.509570 & 0.476852 & -0.193547 \\ 1 & 4.296225 & 0.076846 & 0.450459 \\ 1 & 3.634976 & 0.033335 & -1.179291 \\ 1 & 3.686966 & 1.550023 & -0.290681\end{array}$


Borneol derivative 5 conformer a (1R,2S,4R)-configuration B3PW91/TZ2P optimized geometry [E=-875.830033779 hartrees] Cartesian coordinates (Angstroms)

$\begin{array}{rrrr}6 & 1.216664 & 0.512884 & -0.513477 \\ 6 & 1.432588 & -0.501154 & -1.657385 \\ 6 & 2.056892 & -1.734331 & -0.956925 \\ 6 & 2.075429 & -1.310972 & 0.519511 \\ 6 & 0.614726 & -1.293184 & 0.997076 \\ 6 & 0.032915 & -0.019495 & 0.328343 \\ 6 & 2.421202 & 0.199105 & 0.434559 \\ 8 & -1.116949 & -0.274417 & -0.459492 \\ 14 & -2.675301 & 0.045558 & 0.016188 \\ 6 & -3.744334 & -0.629732 & -1.358386 \\ 1 & -4.803360 & -0.465040 & -1.149064 \\ 1 & -3.510550 & -0.146510 & -2.308591 \\ 1 & -3.588862 & -1.702904 & -1.481514 \\ 6 & -3.065391 & -0.804919 & 1.643273 \\ 1 & -2.417374 & -0.454098 & 2.449313 \\ 1 & -4.096446 & -0.605023 & 1.944460 \\ 1 & -2.944661 & -1.886876 & 1.564690 \\ 6 & -2.937984 & 1.893421 & 0.211670 \\ 1 & -2.724019 & 2.420765 & -0.719754 \\ 1 & -3.973659 & 2.107332 & 0.486268 \\ 1 & -2.300843 & 2.318589 & 0.989819 \\ 1 & -0.215438 & 0.730492 & 1.086446 \\ 1 & 2.097178 & -0.085414 & -2.415911 \\ 1 & 0.487810 & -0.730250 & -2.146927 \\ 1 & 1.467165 & -2.640150 & -1.103621 \\ 1 & 3.061192 & -1.943901 & -1.326851 \\ 1 & 2.741421 & -1.906605 & 1.145913 \\ 1 & 0.533332 & -1.250781 & 2.083885 \\ 1 & 0.066870 & -2.174285 & 0.663017 \\ 6 & 1.037058 & 1.941950 & -0.979727 \\ 1 & 1.895909 & 2.282084 & -1.561756 \\ 1 & 0.905893 & 2.628158 & -0.139728 \\ 1 & 0.154271 & 2.027765 & -1.616217 \\ 6 & 2.389113 & 0.920928 & 1.785172 \\ 1 & 2.500064 & 1.999002 & 1.649132 \\ 1 & 1.479105 & 0.753839 & 2.357965 \\ 1 & 3.225380 & 0.588350 & 2.404424 \\ 6 & 3.787898 & 0.488513 & -0.187649 \\ 1 & 4.581793 & 0.088735 & 0.447623 \\ 1 & 3.914742 & 0.061112 & -1.180293 \\ 1 & 3.952227 & 1.565063 & -0.269758\end{array}$




\section{Synthesis and characterization}

For ${ }^{1} \mathrm{H}(400.13 \mathrm{MHz})$ and ${ }^{13} \mathrm{C}(100.61 \mathrm{MHz})$ NMR spectra, the chemical shifts were related to chloroform.

(+)- and (-)-endo-bornyl acetate, $\mathbf{3}$

(+)-3 was synthesized from (+)-1 (Fluka, puriss. $>99 \%$, er(+):(-)>99:1, $[\alpha]_{D}^{20}=+37$ $(\mathrm{c} 5, \mathrm{EtOH}))$. To a stirred solution of $(+)-\mathbf{1}(9 \mathrm{mmol})$ in $20 \mathrm{~mL}$ of freshly distilled pyridine was added dropwise $25 \mathrm{mmol}(4.2 \mathrm{~mL})$ of acetic anhydride. The mixture was stirred overnight at room temperature. After the addition of $20 \mathrm{~mL}$ water, the mixture was stirred vigorously for $1 \mathrm{~h}$ and then extracted twice with $20 \mathrm{~mL}$ diethyl ether. The organic phase was dried on $\mathrm{MgSO}_{4}$ and the solvent evaporated under vacuum. The residue was purified on a silica gel column. Eluent: Cyclohexane/ethylacetate 9/1. Yield of $(+)-3 \quad 82 \% . R_{f}=0.62 .{ }^{1} \mathrm{H}$ NMR $(400.13 \mathrm{MHz}): \delta: 0.73$ (s, 3H); 0.82 (s, 3H); 0.84 (s, 3H); 0.92 (dd, 1H, J = 3.5 Hz, J =12.5 Hz); 1.19-1.35 (m, 2H); 1.65-1.81 (m, 2H); 1.88-2.00 (m, 1H); $2.06(\mathrm{~s}, 3 \mathrm{H}) ; 2.29-2.42(\mathrm{~m}, 1 \mathrm{H}) ; 4.82-4.92(\mathrm{~m}, 1 \mathrm{H}) ;{ }^{13} \mathrm{C}$ NMR (100.61 MHz): $\delta: 13.5\left(\mathrm{CH}_{3}\right) ; 18.8\left(\mathrm{CH}_{3}\right) ; 19.9\left(\mathrm{CH}_{3}\right) ; 21.3\left(\mathrm{CH}_{3}\right) ; 27.0,28.0(\mathrm{C}-5, \mathrm{C}-6)$; 36.7 (C-3); 44.9 (C-4); 47.7 (C-1); $48.7(\mathrm{C}-7) ; 79.9(\mathrm{C}-2) ; 171.4(\mathrm{CO}) .[\alpha]_{\mathrm{D}}^{25}=+44.8(\mathrm{c} 1.1$, $\mathrm{EtOH})$.

(-)-3 was synthesized from (-)-1, obtained by purification of technical grade (75\%) (-)-1 (Fluka), $[\alpha]_{\mathrm{D}}^{25}=-33.5(\mathrm{c} 2, \mathrm{EtOH})$; ee $90 \%$ by GC. The procedure was the same as for (+)-3. Yield of (-)-3 84\%. Same physical constants and NMR spectra as for (+)-3. $[\alpha]_{D}^{25}=-40.3(\mathrm{c}$ $2.8, \mathrm{EtOH})$.

(+)- and (-)- endo-bornyl-tert-butyl ether, 4.

(+)-4 was synthesized from (+)-1 (Fluka, vide supra), following the procedure of Armstrong et al. [4] To a stirred solution of (+)-1 (5 mmol) in $10 \mathrm{~mL}$ of cyclohexane were added tert-butyl trichloroacetamidate (1.1 eq.) and then a catalytic amount of boron trifluoride etherate $(60 \mu \mathrm{L})$. The mixture was stirred at room temperature for 24 hours. After the addition 
of solid $\mathrm{NaHCO}_{3}(1 \mathrm{~g})$, the mixture was stirred vigorously for 30 minutes, filtered and the filtrate was evaporated under vacuum. The crude product was purified on a silica gel column. Eluent: Cyclohexane/Ethylacetate 7/3. Yield of (+)-4 75\%. $\mathrm{R}_{\mathrm{f}}=0.8 .{ }^{1} \mathrm{H}$ NMR (400.13 MHz): $\delta: 0.70$ (s, 3H); 0.76 (s, 3H); 0.78 (s, 3H); 0.86 (dd, 1H, J = 3.6 Hz, J =13.2 Hz); 1.05 (s, 9H); 1.11-1.21 $(\mathrm{m}, 2 \mathrm{H}) ; 1.48-1.63(\mathrm{~m}, 2 \mathrm{H}) ; 1.89-2.07(\mathrm{~m}, 2 \mathrm{H}) ; 3.54-3.61(\mathrm{~m}, 1 \mathrm{H}) ;{ }^{13} \mathrm{C}$ NMR $(100.61 \mathrm{MHz}): \delta$ : $13.5\left(\mathrm{CH}_{3}\right) ; 18.9\left(\mathrm{CH}_{3}\right) ; 19.9\left(\mathrm{CH}_{3}\right) ; 26.5,28.3(\mathrm{C}-5, \mathrm{C}-6) ; 28.6\left(\left(\mathrm{CH}_{3}\right)_{3}\right) ; 40.0(\mathrm{C}-3) ; 45.4(\mathrm{C}-4)$; $47.0(\mathrm{C}-1) ; 48.9(\mathrm{C}-7) ; 72.2(\mathrm{C}-9-t-\mathrm{Bu}) ; 75.8(\mathrm{C}-2) .[\alpha]_{\mathrm{D}}^{25}=+50.4\left(\mathrm{c} 1.5, \mathrm{CHCl}_{3}\right)$.

(-)-4 was synthesized from (-)-1 (Fluka, purum 97\%, ee $96 \%$ by GC, $[\alpha]_{D}^{20}=-36$ (c 2.5, EtOH)). The procedure was the same as for (+)-4. Yield of (-)-4 74\%. Same physical constants and NMR spectra as for (+)-4. $[\alpha]_{\mathrm{D}}^{25}=-48.7\left(\mathrm{c} 1.3, \mathrm{CHCl}_{3}\right)$.

(+)- and (-)- endo-bornyl-trimethylsilyl ether, $\mathbf{5}$

(+)-5 was synthesized from (+)-1 (Fluka, vide supra). To a stirred solution of (+)-1 (10 $\mathrm{mmol})$ in $20 \mathrm{~mL}$ of anhydrous THF was added freshly distilled triethylamine (20 mmol, $2 \mathrm{~g} ; 2.8$ $\mathrm{mL})$, and then dropwise a solution of trimethylsilyl chloride $(1.3 \mathrm{~g}, 12.1 \mathrm{mmol})$ in $10 \mathrm{~mL}$ of anhydrous THF. The mixture was stirred overnight. A white precipitate appeared. After the concentration of the mixture under vacuum, the residue was dissolved in $70 \mathrm{~mL}$ of ether, and the organic phase was washed three times with water. The organic phase was dried on $\mathrm{MgSO}_{4}$ and the solvent evaporated under vacuum. The crude product was purified on a silica gel column. Eluent: Cyclohexane. Yield of (+)-5 75\%. $\mathrm{R}_{\mathrm{f}}=0.95 .{ }^{1} \mathrm{H}$ NMR (400.13 MHz): $\delta: 0.00(\mathrm{~s}, 9 \mathrm{H})$; $0.70(\mathrm{~s}, 3 \mathrm{H}) ; 0.78(\mathrm{~s}, 3 \mathrm{H}) ; 0.80(\mathrm{~s}, 3 \mathrm{H}) ; 0.92(\mathrm{dd}, 1 \mathrm{H}, \mathrm{J}=3.5 \mathrm{~Hz}, \mathrm{~J}=12.5 \mathrm{~Hz}) ; 1.01-1.22(\mathrm{~m}, 2 \mathrm{H})$; $1.48-1.58(\mathrm{~m}, 1 \mathrm{H}) ; 1.58-1.69(\mathrm{~m}, 1 \mathrm{H}) ; 1.89-2.02(\mathrm{~m}, 1 \mathrm{H}) ; 2.02-2.18(\mathrm{~m}, 1 \mathrm{H}) ; 3.78-3.85(\mathrm{~m}, 1 \mathrm{H})$; ${ }^{13} \mathrm{C}$ NMR (100.61 MHz): $\delta: 0.0\left(\left(\mathrm{CH}_{3}\right)_{3}\right) ; 11.7\left(\mathrm{CH}_{3}\right) ; 20.0\left(\mathrm{CH}_{3}\right) ; 20.5\left(\mathrm{CH}_{3}\right) ; 27.3,33.9(\mathrm{C}-5$, C-6); 42.1 (C-3); $45.2(\mathrm{C}-4) ; 46.4(\mathrm{C}-1) ; 48.8(\mathrm{C}-7) ; 79.4(\mathrm{C}-2) .[\alpha]_{\mathrm{D}}^{25}=+43.8$ (c $1.3, \mathrm{CHCl}_{3}$ ).

(-)-5 was synthesized from (-)-1 (obtained as in the synthesis of (-)-3). The procedure was the same as for (+)-5. Yield of (-)-5 74\%. Same physical constants and NMR spectra as for (+)-5. $[\alpha]_{\mathrm{D}}^{25}=-38.9\left(\mathrm{c} 1.3, \mathrm{CHCl}_{3}\right)$. 
For IR and VCD spectroscopy, (+)- and (-)-1 were from Aldrich: (+)-1, $[\alpha]_{\mathrm{D}}{ }^{20}+36(\mathrm{c} 5$, $\mathrm{EtOH}) ;(-)-1,[\alpha]_{\mathrm{D}}{ }^{20}-35.6(\mathrm{c} 5, \mathrm{EtOH})$.

The Absolute Configuration (AC) of $\mathbf{1}$ is $1 \mathrm{R}, 2 \mathrm{~S}, 4 \mathrm{R}-(+) / 1 \mathrm{~S}, 2 \mathrm{R}, 4 \mathrm{~S}-(-)$. Since (+)-1 gives (+)-3, (+)-4 and (+)-5, it follows that the ACs of 3, $\mathbf{4}$ and $\mathbf{5}$ are the same as the AC of $\mathbf{1}$. 